\title{
ON THE GROWTH OF TAYLOR-GÖRTLER VORTICES ALONG HIGHLY CONCAVE WALLS*
}

\author{
BY \\ A. M. O. SMITH \\ Douglas Aircraft Company, Inc., El Segundo, Calif.
}

1. Abstract. The primary objective of this study has been to prepare a chart for computing the growth of Taylor-Görtler vortices in laminar flow along walls of both high and low concave curvature. Taylor-Görtler vortices are streamwise vortices having alternate right- and left-hand rotation that may develop in the laminar boundary layer along a concave surface.

The equations of motion are derived anew and re-examined with regard to the importance of the various terms. The final equations used in preparation of the chart are found to be valid for radii of curvature as small as $\mathbf{3 0}$ times the boundary layer thickness. Furthermore, it is shown that the equations are not restricted in validity to cases of constant wall curvature, constant free stream velocities, or to boundary layers of constant thickness. Whereas the previous analyses by Taylor and Görtler assumed the vortex to grow exponentially as a function of time, the present study recasts the growth into a more convenient form in which the vortex grows as a function of distance.

The solution is an eigenvalue problem, which in the present study has been solved mainly by Galerkin's method-a variational method. Both the eigenvalues and the eigenfunctions are presented, the former in the aforementioned chart. It is possible to compare the solutions for neutral stability with those given by Görtler. The two solutions are in approximate agreement.

A second method of solution also is described. This method is believed to offer considerable improvement, provided a high-speed digital computer is available. In the one case checked by both methods agreement was within $2 \%$.

Finally, the stability chart was applied to all the known experimental data concerning the effect of concave curvature on the transition point. The well known parameter $R_{\theta}(\theta / r)^{1 / 2}$ is shown to be inadequate as an indicator of the transition point. Instead, the experimental data indicate that an apparent amplification factor, $\exp \int \beta d x$, is a much better measure. Available results show that transition of this type will occur when $\int \beta d x$ reaches a value of about ten.

2. The flow past a concave plate. A considerable body of indirect evidence indicates that a laminar flow along a concave wall does not remain two-dimensional. Instead, it rolls up into alternate right- and left-hand vortices as indicated in Fig. 1. To obtain some insight into the forces that cause the formation of these vortices, consider the streaming of an incompressible, viscous fluid past a concave wall, Fig. 2 . If the Reynolds number is not extremely low, a boundary layer will develop.

At some arbitrary height, $y_{1}$, within the boundary layer, the velocity is $u_{1}$. At some other height $y_{2}=y_{1}+\Delta y$ the velocity is $u_{2}=u_{1}+(\partial u / \partial y) \Delta y$. At the height $y_{1}$, the fluid is under a pressure $p_{1}$. By the usual boundary layer equations for two-dimensional

\footnotetext{
*Received August 30, 1954; revised manuscript received December 24, 1954. This paper is a condensation of Douglas Report E.S. 17110 dated 3 March 1953 which see for more complete details concerning the analysis and numerical methods.
} 


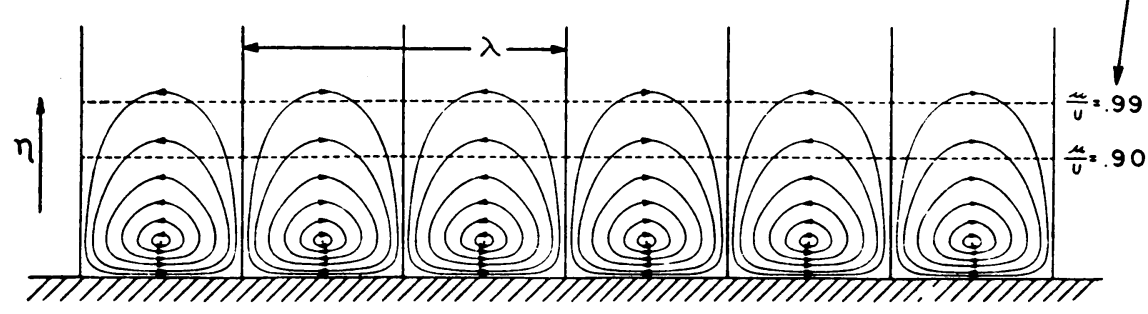

SA) STRONG INSTABILITY, HIGH CURVATURE ( $A=1, B R=200)$

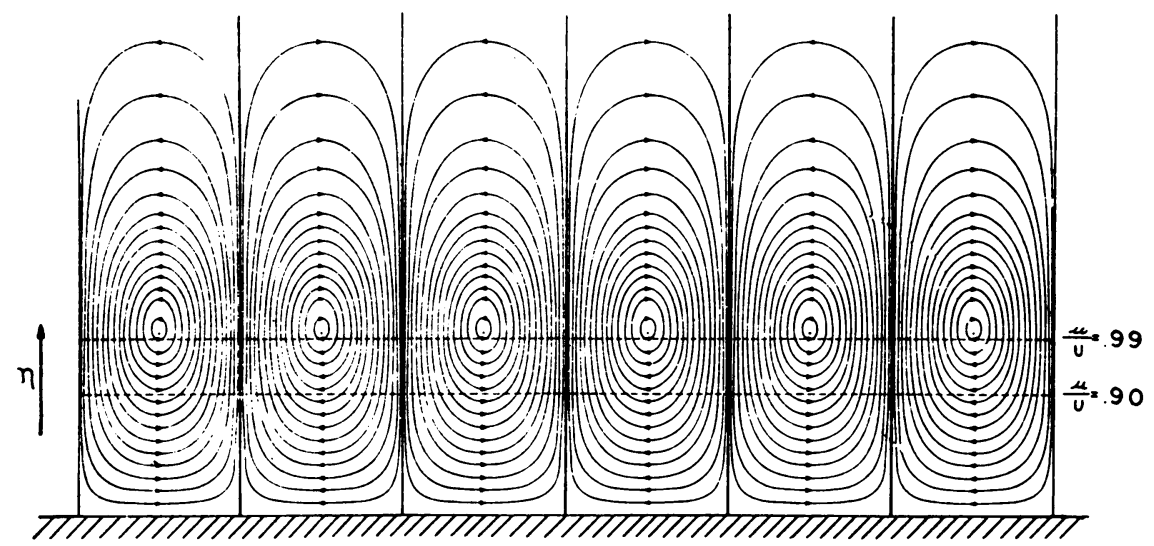

(B) NEUTRAL STABILITY, SLIGHT CURVATURE $(A=1, B R=0)$

Fig. 1. Streamline patterns for one width of vortex as calculated for walls of high and low concave curvature. View looking directly upstream or downstream.

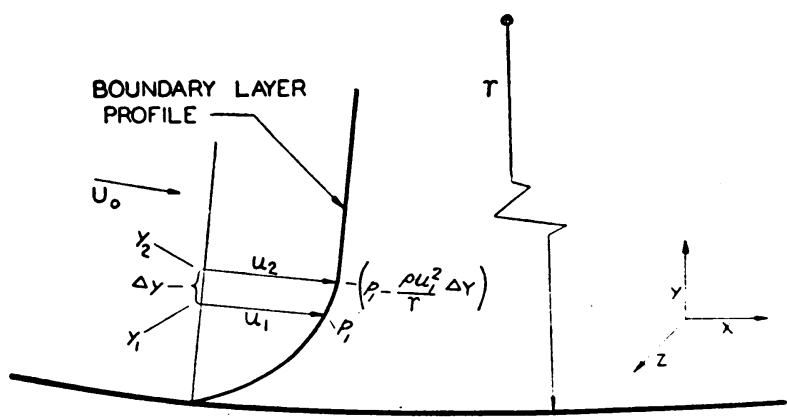

Fig. 2. Boundary layer flow along a curved plate. 
curved flow, (for instance, Eq. 8, page 120, Ref. [1]) the steady state pressure at $y_{2}$ is $p_{1}-(\rho / r) u_{1}^{2} \Delta y$.

Suppose an element of fluid from the position $y_{1}$ is displaced outwards, as by a swirl, until it reaches the height $y_{2}$. If there is negligible energy dissipation, the element will not lose any total head, so that with the pressure existing at $y_{2}$, a new velocity $u_{2}^{\prime}$ will be obtained as follows:

$$
\begin{aligned}
p_{1}+\frac{1}{2} \rho u_{1}^{2} & =p_{2}+\frac{1}{2} \rho u_{2}^{\prime 2} \\
& =p_{1}-r^{-1} \rho u_{1}^{2} \Delta y+\frac{1}{2} \rho u_{2}^{\prime 2}
\end{aligned}
$$

or

$$
u_{2}^{\prime}=u_{1}(1+2 \Delta y / r)^{1 / 2} .
$$

Expanding, the inviscid change in velocity, $u_{2}^{\prime}-u_{1}$, to first order is $u_{1}(\Delta y / r)$.

In the basic boundary layer the change in velocity accompanying a change $\Delta y$ is $u_{2}-u_{1}=(\partial u / \partial y) \Delta y$. Thus the change $u_{2}^{\prime}-u_{1}$ is smaller than $u_{2}-u_{1}$ by the order of $\delta / r$ where $\delta$ is the boundary layer thickness. The displaced element of fluid will now be out of balance with its surroundings because of centrifugal force. If the element has been displaced outwards, the centrifugal effect will give it a sort of buoyancy that enables it to continue its movement. If displaced inwards from its position of equilibrium, it will tend to move right to the wall, for its centrifugal force will exceed that of the surrounding fluid. Thus, in the case of concave flow, a condition of instability exists. The situation is somewhat similar to that occurring when a body of fluid rests over a hot surface.

Such a condition of instability in the flow has been recognized since early in this century, Lord Rayleigh [2] being one of the first to study it. His studies, however, were concerned only with the flow between rotating concentric cylinders. Apparently, until G. I. Taylor [3] tackled the problem no one had conceived the type of motion that this instability would produce. Taylor assumed it would produce vortices in the boundary layer which would trail in the direction of flow. In some respects, they are similar to wing trailing vortices except that they have alternate right- and left-hand rotation. Taylor formulated the motion in mathematical terms, analyzed its stability and verified the analysis experimentally in quite conclusive fashion. However, his attentions were concentrated entirely on the problem of flow between rotating concentric cylinders.

The first investigation of the vortex mode of motion in a boundary layer was carried out by Görtler [4] at the Kaiser Wilhelm Institute under Prandtl. Görtler's analysis was exploratory in approach, for, never having been made before, there was no evidence that this type of flow could exist in a boundary layer. In fact, one of the reasons for the investigation was that the group at Göttingen was exploring all avenues that might assist in explaining the onset of turbulence. Görtler's analysis produced a parameter that served as a measure of the instability of the flow, namely, $R_{\theta}(\theta / r)^{1 / 2}$ where $R_{\theta}$ is the boundary layer Reynolds number $\left(U_{0} \theta / \nu\right), \theta$ the momentum thickness and $r$ the radius of curvature of the plate. Görtler performed no experiments to verify his analysis. Subsequently Liepmann [5] at the California Institute of Technology ran tests which did verify Görtler's analysis that $R_{\theta}(\theta / r)^{1 / 2}$ was a controlling parameter. Liepmann's tests were confined to measuring the effect of curvature on transition, no effort being made to ascertain whether vortices of the type assumed in the mathematics did exist 
in fact. Nevertheless, the experimental data point very strongly to the existence of this type of vortex flow in the boundary layer. In retrospect:

(a) Taylor and Hagerty [6] show photographs of this type of vortex flow between rotating cylinders.

(b) The stability, size, and shape of the vortices in (a) agree excellently with calculations.

(c) The theoretical parameter $R_{\theta}(\theta / r)^{1 / 2}$ characterizing vortex flow in the boundary layer of a concave wall, was demonstrated by Liepmann to be valid.

3. Previous theoretical investigations. Taylor's original analysis in 1923 is not applicable to the problem of flow in a boundary layer, for his work dealt with the stability of viscous flow between concentric rotating cylinders. Furthermore, his equations are formulated in terms of cylindrical coordinates. Several additional theoretical attacks on the same problem were made by other investigators, but not until 1940 was the same mode of disturbance studied for ordinary boundary layer flow-by Görtler.

Görtler's objective was primarily one of learning whether such a type of motion could be unstable. Therefore, to seek the essence of the problem he analyzed only the case of weak instability, for his major concern was the prediction of neutral stability. As a consequence, he was able to drop a large number of terms in the equations and produce a conveniently tractable expression which clearly indicated the dominant parameters. The equations form a sixth order system, but fortunately, they lent themselves well to solution of the eigenvalue problem by means of integral equations using Green's functions. Görtler's work did show that an unstable vortex flow within the boundary layer could exist, but only for plates with concave curvature. His studies indicated that the stability is not very sensitive to the shape of the boundary layer (contrast with Tollmien-Schlichting type of disturbance).

The vortices under consideration represent a steady type of flow. That is, if the vortices were restrained from wandering back and forth in a spanwise direction, a hot wire fixed on the model would show a steady reading. Thus, such a disturbance is quite different from the Tollmien-Schlichting type which produces a wavelike response on the hot wire. Accordingly, it is most logical and nearest to the physical picture to assume that these vortices grow with distance. Görtler, however, as well as Taylor, analyzed a disturbance that grew in time. For their purposes a temporal variation was satisfactory, because the neutral stability condition was the focal point of their work. Obviously, either basis is satisfactory for the determination of neutral stability, because a disturbance that does not grow with time cannot grow with distance. Nevertheless, strictly speaking, a disturbance assumed to be growing in time implies that a hot wire fixed on the surface would show an exponential growth of signal. When dealing with growing boundary layers in a variable velocity field, Görtler's method becomes untenable, for derivatives with respect to both $x$ and $t$ will exist. In the simpler case of parallel flows, the $x$-derivatives either equal zero or are negligible.

More recently Meksyn [7] solved anew both Taylor's and Görtler's problems by the method of asymptotic expansions. For the problem of stability of motion between two rotating cylinders, his results verify Taylor's calculations, but are obtained by simpler formulae, demanding far less labor to evaluate.

In the case of boundary layer flow, since he begins with Görtler's differential equa- 
tions, the problem treated is the limited mathematical one of finding a better and less laborious solution to a given differential equation. However, Meksyn's determination. of the neutral stability limit disagrees considerably with Görtler's value, whereas, the results of the present work, obtained by a third and fourth method, agree satisfactorily with Görtler's values. Unfortunately, time was not available to the author to learn the cause for Meksyn's different result*.

4. Purpose of the present work. Regions of concave curvature exist in a number of aerodynamic applications. Compressor blading, turbine blading, and turning vanes are examples of one type. Another type, but beyond the scope of this work, is the isentropic spike diffuser. A third class of examples exists in boundary layer control where velocity discontinuities are used, for immediately behind a discontinuity there exists a region of strong concave curvature. Griffith airfoils are shapes on which such large concave regions occur.

In a special application, regions of strong concave curvature existed, but for very short distances. There was a good chance that even though the flow was strongly unstable, the instability did not last long enough for Görtler vortices to grow sufficiently to cause transition. Bearing in mind that the work of Görtler, Liepmann, and Taylor proves that such vortices dominate the flow in regions of sufficient concave curvature, the problem at hand justified recalculation of the stability of these vortices for large amounts of curvature. The objective was to prepare a chart for use in routine computation. As only the region of least stability was of interest, no new statements about the mathematical properties of the solution can be made. For information regarding the asymptotic nature of the solution, the number of modes in which this instability may occur, etc., the reader is referred to the articles by Görtler, Meksyn, and Hämmerlin. Some caution should be used, however, in examining their limiting solutions in view of the fact that they are obtained from greatly simplified equations.

5. Method of attack. The problem posed is an eigenvalue problem of caleulating the rate of growth of Görtler vortices. It is assumed that the vortices never reach great strength. Thus, the equations can be linearized by the usual methods of small disturbance theory. But it is not assumed that the rate of growth is small; in fact, the equations are written to treat cases of great rates of growth. In developing the equations, therefore, many terms had to be retained that Görtler discarded. This action so complicates the equations, that solutions using either Görtler's or Meksyn's method did not seem possible.

At this point a decision became necessary, for two courses were possible: (a) to discard terms until the equations were simple enough to solve by theoretically exact methods, that is, to secure "exact" solutions of an approximate equation, or (b) to retain the second order terms and solve by approximate methods, that is, to secure approximate solutions of an "exact" equation. The second choice was taken in the belief that even the "exact" methods used by Görtler and Meksyn required many approximations, even to solve their simpler problems. In particular, the question of accuracy seemed more easily answerable by using the second course. For instance, the effect of discarded terms is hard to visualize, even when a simple analytic solution is obtainable. However,

${ }^{*}$ However, the discrepancy as well as the mathematical details of both methods have recently been examined in a Doctoral Dissertation by Günther Hämmerlin [13]. In general, Hämmerlin agrees with Görtler’s results. 
if approximate methods are used for solving an exact equation, it is certain that the equation itself contributes no error. The error contributed by the approximate method of solution can usually be sensed rather easily by making a few convergence studies.

Of the several approximate methods available, Galerkin's method appeared most promising. It was used successfully, and appears to have given the desired accuracy. Galerkin's method is so powerful for handling linear equations, that the complexity or number of terms has only a minor effect on the total number of man-hours required to obtain an answer. Consequently, once Galerkin's method was forced by requirements of the problem, the incentive to eliminate all terms of secondary importance disappeared. Therefore, in the final equations, only terms of extremely minor effect have been discarded. However, the work was most laborious, for every eigenvalue required the solution of a twelfth order system of simultaneous equations, including the inversion of a difficult twelfth order matrix.

Near the end of the work another method, a much more powerful and more exact method, was developed. This second method requires very little labor to set up a case. If a high speed digital calculator is available, the remaining labor is trivial. Without such a machine, this second method would be even more laborious than Galerkin's. The method is an iterative interpolation procedure that will be described in Sec. 14 .

6. Transformation of the Navier-Stokes equation into a system using curvilinear coordinates. Notation in the subsequent sections in general follows that of Refs. [1] and [4]. We shall consider only the case of two-dimensional flow. The basic flow is two-dimensional, but within it will be developed the three-dimensional disturbance. Since the problem under consideration deals with flows along walls of variable curvature, a coordinate system both convenient and capable of accommodating itself to the necessary arbitrary boundaries is an orthogonal system of "parallel" curves. Because the base motion is two-dimensional, the coordinate system will have curvature in only one direction, and the wall itself will be of cylindrical shape. Generatrices of the surface will be at right angles to the plane of the two-dimensional flow. Figure 3 illustrates the

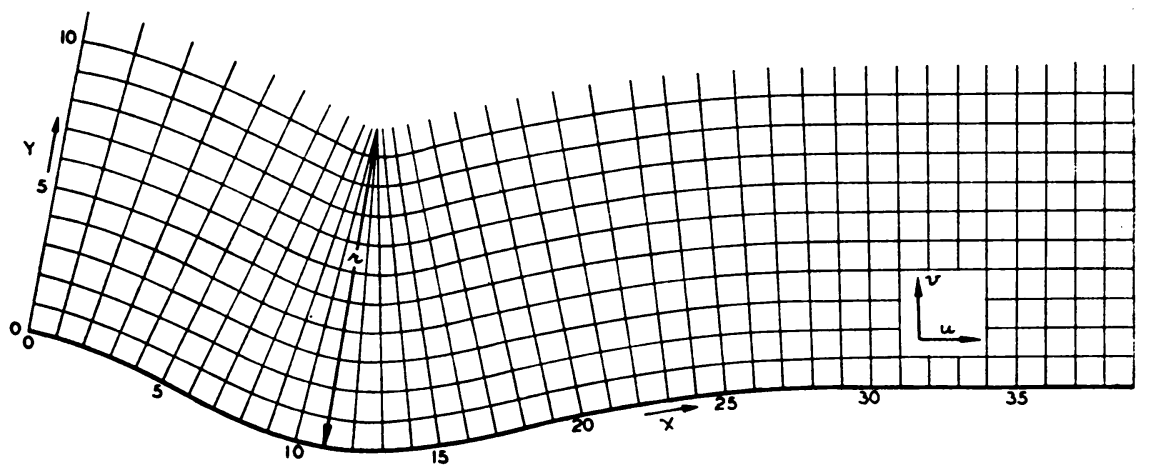

FIg. 3. The parallel curve system of orthogonal curvilinear coordinates.

coordinate system. The line $y=0$ coincides with the curved wall and the $z$-direction is perpendicular to the plane of the paper. In the present problem the top of the boundary layer for the most extreme curvature considered would occur about at $y=1$ in Fig. 3. Therefore, no difficulties with singularities will occur. 
In vector notation the steady state incompressible form of the full Navier-Stokes equation is: (Eq. 30, page 100, Ref. [1])

$$
\nabla \times \omega=\operatorname{grad}\left(\frac{p}{\rho}+\frac{1}{2} \nabla^{2}\right)+\nu \operatorname{curl} \omega .
$$

Following Goldstein, this equation can be translated into the curvilinear coordinate system. The transformation differs in only one respect from that outlined by Goldstein. He assigns a negative sign to concave curvature. In the present analysis, since concave curvature is the center of interest, it is given a positive sign.

The complete, exact Navier-Stokes equations for steady, incompressible flow along a boundary having a curvature $\kappa \equiv 1 / r$ are found to be:

$$
\left.\begin{array}{c}
\frac{1}{1-\kappa y} u \frac{\partial u}{\partial x}+v \frac{\partial u}{\partial y}+w \frac{\partial u}{\partial z}-\frac{\kappa u v}{1-\kappa y}=\frac{-1}{\rho(1-\kappa y)} \frac{\partial p}{\partial x} \\
+\nu\left[\nabla^{2} u-\frac{2 \kappa}{(1-\kappa y)^{2}} \frac{\partial v}{\partial x}-\frac{\kappa^{2} u}{(1-\kappa y)^{2}}-\frac{\kappa}{1-\kappa y} \frac{\partial u}{\partial y}-\frac{v(d \kappa / d x)}{(1-\kappa y)^{3}}\right] \\
\frac{1}{1-\kappa y} u \frac{\partial v}{\partial x}+v \frac{\partial v}{\partial y}+w \frac{\partial v}{\partial z}+\frac{\kappa u^{2}}{1-\kappa y}=-\frac{1}{\rho} \frac{\partial p}{\partial y} \\
+\nu\left[\nabla^{2} v+\frac{2 \kappa}{(1-\kappa y)^{2}} \frac{\partial u}{\partial x}-\frac{\kappa^{2} v}{(1-\kappa y)^{2}}-\frac{\kappa}{1-\kappa y} \frac{\partial v}{\partial y}+\frac{u(d \kappa / d x)}{(1-\kappa y)^{3}}\right] \\
\frac{1}{1-\kappa y} u \frac{\partial w}{\partial x}+v \frac{\partial w}{\partial y+w \frac{\partial w}{\partial z}=-\frac{1}{\rho} \frac{\partial p}{\partial z}+\nu\left[\nabla^{2} w-\frac{\kappa}{1-\kappa y} \frac{\partial w}{\partial y}\right]} \\
\operatorname{Div} \mathbf{v} \equiv \nabla \cdot \nabla=\frac{1}{1-\kappa y} \frac{\partial u}{\partial x}+\frac{\partial v}{\partial y}+\frac{\partial w}{\partial z}-\frac{\kappa v}{1-\kappa y}=0 \\
\text { where } \quad \nabla^{2} \equiv \frac{1}{(1-\kappa y)^{2}} \frac{\partial^{2}}{\partial x^{2}}+\frac{\partial^{2}}{\partial y^{2}}+\frac{\partial^{2}}{\partial z^{2}}+\frac{y(d \kappa / d x)}{(1-\kappa y)^{3}} \frac{\partial}{\partial x} \cdot
\end{array}\right\}
$$

7. Mathematical description of the disturbance. Görtler, following the work of Taylor, described the vortex motion in the boundary layer by means of the following equations, in which all values are real numbers.

$$
\begin{aligned}
& u=u_{0}(y)+u_{1}(y) \cos \alpha z \exp \beta t, \\
& v=\quad v_{1}(y) \cos \alpha z \exp \beta t, \\
& w=\quad w_{1}(y) \sin \alpha z \exp \beta t, \\
& p=p_{0}(y)+p_{1}(y) \cos \alpha z \exp \beta t .
\end{aligned}
$$

In the above: $u, v$ and $w$ are components of the entire velocity as already used; $u_{0}$ represents the undisturbed velocity that would exist for the same flow conditions on a plate without curvature; $p_{0}$ likewise represents the basic, undisturbed pressure; $u_{1}, v_{1}, w_{1}, p_{1}$ are the superimposed disturbance functions; $\alpha$ is a measure of the width of the vortex; $\alpha=2 \pi / \lambda$ where $\lambda$ is the wave length (see Fig. 1); and $\beta$ is a measure of the rate of growth of the disturbance as a function of time. As these equations show, Görtler treated the simplified case of constant velocity parallel flows, for $v_{0}=0$, and $u_{0}$ and $p_{0}$ are independent 
of $x$. Moreover, as brought out earlier, the disturbance grows in time proportional to the term $\exp \beta t$, or, if the equations were to be interpreted literally, the expressions (4) state that at one $x, y$ and $z$ location in a steady base flow, the disturbance is changing strength in an exponential fashion. Furthermore, the term exp $\beta t$ implies that $\beta$ is invariant with time, that is, the radius of curvature must be constant.

Part of the purpose of the present analysis is to learn the importance of previously neglected terms in concave flows where both the radius of curvature and boundary layer thickness may be functions of $x$. Therefore, the stability of vortices in a more general flow will be studied by considering the type of disturbance described below, in which $\beta$ now has a new meaning:

$$
\begin{aligned}
& u(x, y)=u_{0}(x, y)+u_{1}(y) \cos \alpha z \exp \int \beta(x) d x, \\
& v(x, y)=v_{0}(x, y)+v_{1}(y) \cos \alpha z \exp \int \beta(x) d x, \\
& w(x, y)=0+w_{1}(y) \sin \alpha z \exp \int \beta(x) d x, \\
& p(x, y)=p_{0}(x, y)+p_{1}(y) \cos \alpha z \exp \int \beta(x) d x .
\end{aligned}
$$

Equations (5) assume that $u_{0}$ and $p_{0}$ may be functions of $x$, and also assume a vertical component, $v_{0}$, of the basic velocity. The vortex is assumed to grow in strength by the factor $\exp \int \beta d x$, that is, to grow with distance, not time. Furthermore, if wall curvature varies, $\beta$ would not remain constant, that is, $\beta$ varies with $x$. For this reason, the simpler form used by Görtler is replaced by an integral.

In generalizing the earlier form, an inconsistency is introduced. If, as in Görtler's analysis the base flow is strictly a constant velocity, parallel type along a wall of constant curvature, then $u_{1}, v_{1}$ and $w_{1}$ are truly functions of $y$ only. However, when, for instance, the curvature (and hence $\beta$ ) changes, the eigenfunctions $u_{1}, v_{1}$ and $w_{1}$ will change. It follows then that these disturbance functions are not strictly functions of $y$ only. Nevertheless, the assumption of independence of $x$ is not believed to introduce appreciable error, for the functions $u_{1}(y)$, etc., vary only slightly with $\beta$. Furthermore, it is shown later that $\partial u_{0} / \partial x, \partial v_{0} / \partial x, \partial \kappa / \partial x, \partial \beta / \partial x, \partial p_{0} / \partial x$ are distinctly minor factors compared with the major terms in the equations. This observation is further evidence that neglect of variations with $x$ of $u_{1}, v_{1}$, etc., has an insignificant effect on the solutions.

8. Development of the differential equations. Expressions (5) can be differentiated, as necessary and substituted into Eqs. (2). Although the degree of instability may be great, the strength of the disturbance is assumed to be small. Therefore, products and squares of the disturbance will be neglected with respect to products of the disturbance times base flow values; in other words, the conventional methods of linearization will be followed. After the substitutions have been made, all base flow terms can be eliminated by subtracting Eqs. (2), which, of course, apply equally well to the undisturbed flow. Then we are left with equations from which the factor $\exp \int \beta d x \cos \alpha z$ or $\exp \int \beta d x$ $\sin \alpha z$ can be extracted and cancelled out to leave a set of ordinary simultaneous differential equations in $u_{1}, v_{1}, w_{1}$ and $p_{1}$, namely, Eqs. (6) and (7) 


$$
\begin{aligned}
& \nu \frac{d^{2} u_{1}}{d y^{2}}-\left(v_{0}+\frac{\kappa \nu}{1-\kappa y}\right) \frac{d u_{1}}{d y}+\left[\frac{\nu}{(1-\kappa y)^{2}} \frac{d \beta}{d x}+\frac{\beta^{2} \nu}{(1-\kappa y)^{2}}-\alpha^{2} \nu\right. \\
& \left.+\frac{\beta y \nu}{(1-\kappa y)^{3}} \frac{d \kappa}{d x}-\frac{\kappa^{2} \nu}{(1-\kappa y)^{2}}-\frac{\beta u_{0}}{1-\kappa y}+\frac{\partial v_{0}}{\partial y}\right] u_{1}-\left[\frac{\partial u_{0}}{\partial y}-\frac{\kappa u_{0}}{1-\kappa y}\right. \\
& \left.+\frac{2 \beta \kappa \nu}{(1-\kappa y)^{2}}+\frac{\nu}{(1-\kappa y)^{3}} \frac{d \kappa}{d x}\right] v_{1}=\frac{\beta p_{1}}{\rho(1-\kappa y)} \\
& \nu \frac{d^{2} v_{1}}{d y^{2}}-\left(v_{0}+\frac{\kappa \nu}{1-\kappa y}\right) \frac{d v_{1}}{d y}+\left[\frac{\nu}{(1-\kappa y)^{2}} \frac{d \beta}{d x}+\frac{\beta^{2} \nu}{(1-\kappa y)^{2}}-\alpha^{2} \nu\right. \\
& \left.+\frac{\beta y \nu}{(1-\kappa y)^{3}} \frac{d \kappa}{d x}-\frac{\kappa^{2} \nu}{(1-\kappa y)^{2}}-\frac{\beta u_{0}}{1-\kappa y}-\frac{\partial v_{0}}{\partial y}\right] v_{1}-\left[\frac{1}{1-\kappa y} \frac{\partial v_{0}}{\partial x}+\frac{2 \kappa u_{0}}{1-\kappa y}\right. \\
& \left.-\frac{2 \beta \kappa \nu}{(1-\kappa y)^{2}}-\frac{\nu}{(1-\kappa y)^{3}} \frac{d \kappa}{d x}\right] u_{1}=\frac{1}{\rho} \frac{d p_{1}}{d y} \\
& \nu \frac{d^{2} w_{1}}{d y^{2}}-\left(v_{0}+\frac{\kappa \nu}{1-\kappa y}\right) \frac{d w_{1}}{d y}+\left[\frac{\nu}{(1-\kappa y)^{2}} \frac{d \beta}{d x}+\frac{\beta^{2} \nu}{(1-\kappa y)^{2}}-\alpha^{2} \nu\right. \\
& \left.+\frac{\beta y \nu}{(1-\kappa y)^{3}} \frac{d \kappa}{d x}-\frac{\beta u_{0}}{1-\kappa y}\right] w_{1}=-\frac{\alpha p_{1}}{\rho} \\
& \frac{1}{1-\kappa y} \beta u_{1}+\frac{d v_{1}}{d y}+\alpha w_{1}-\frac{\kappa v_{1}}{1-\kappa y}=0 \quad \text { (continuity). }
\end{aligned}
$$

In the development of the equations, $(1-\kappa y)^{-1} \partial u_{0} / \partial x$ has been eliminated by means of Eq. (3) applied to the base flow. Other than for the aforementioned approximations, these equations are exact. The relative importance of the various terms next must be examined. It is more satisfactory to make this examination, however, after the equations have been transformed into a non-dimensional system.

9. Reduction to non-dimensional form. Beyond this point no further use of the original Navier-Stokes equation is needed. Therefore, there should be no conflict in notation if we replace $u_{1}$ by $U_{0} u$, etc. In particular let

$U_{0}=$ free stream base velocity outside the boundary layer

$\delta=$ a characteristic measure of the thickness of the boundary layer

$c=$ a measure of the length in the $x$ direction, characteristically a chord length

Then we can write

$$
\begin{aligned}
& R=\frac{U_{0} \delta}{\nu}, \quad u_{0}=U U_{0}, \quad v_{0}=V U_{0}, \quad w_{0}=0 ; \\
& B=\beta \delta, \quad u_{1}=u U_{0}, \quad v_{1}=v U_{0}, \quad w_{1}=w U_{0} ; \\
& A=\alpha \delta, \quad y=\eta \delta, \quad p_{1}=C_{p} \cdot \frac{1}{2} \rho U_{0}^{2} ; \\
& K=\kappa \delta, \quad \frac{d u_{1}}{d y}=\frac{U_{0}}{\delta} \frac{d u}{d \eta}, \quad \frac{d^{2} u_{1}}{d y^{2}}=\frac{U_{0}}{\delta^{2}} \frac{d^{2} u}{d \eta^{2}}, \quad \frac{d \kappa}{d x}=\frac{1}{c^{2}} \frac{d(c \kappa)}{d(x / c)}, \quad \text { etc. }
\end{aligned}
$$

The vertical dimension chosen as characteristic of the boundary layer thickness is that used in the Falkner-Skan boundary layer equation, Sec. 54, Ref. [1] and illustrated in 
Fig. 4. Using this system if $\delta$ corresponds to $Y=1$, then $\eta \equiv Y$. However, to be consistent with Görtler, $\eta$ will continue to be used. Notice further that if the base flow is one of the Falkner-Skan family, $d V / d \eta$, etc. are functions of $\eta$ only, not $x$.

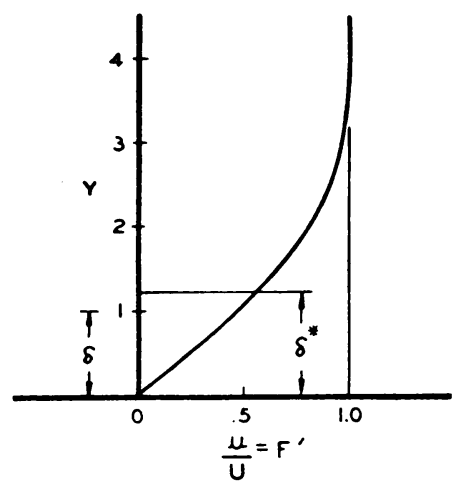

Fig. 4.

Upon making the above substitutions, Eqs. (6) and (7) are transformed into Eqs. (8) and (9).

$$
\begin{aligned}
\frac{d^{2} u}{d \eta^{2}}- & {\left[R V+\frac{K}{1-K \eta}\right] \frac{d u}{d \eta}+\left[\frac{(\delta / c)}{(1-K \eta)^{2}} \frac{d B}{d(x / c)}+\frac{B^{2}}{(1-K \eta)^{2}}-A^{2}\right.} \\
+ & \left.\frac{\left(\delta^{2} / c^{2}\right) B \eta}{(1-K \eta)^{3}} \frac{d(c \kappa)}{d(x / c)}-\frac{K^{2}}{(1-K \eta)^{2}}-\frac{B R U}{1-K \eta}+R \frac{d V}{d \eta}\right] u \\
- & {\left[R \frac{d U}{d \eta}-\frac{K R U}{1-K \eta}+\frac{2 B K}{(1-K \eta)^{2}}+\frac{\left(\delta^{2} / c^{2}\right)}{(1-K \eta)^{3}} \frac{d(c \kappa)}{d(x / c)}\right] v=\frac{B R C_{p}}{2(1-K \eta)} } \\
\frac{d^{2} v}{d \eta^{2}}- & {\left[R V+\frac{K}{1-K \eta}\right] \frac{d v}{d \eta}+\left[\frac{(\delta / c)}{(1-K \eta)^{2}} \frac{d B}{d(x / c)}+\frac{B^{2}}{(1-K \eta)^{2}}-A^{2}\right.} \\
+ & \left.\frac{\left(\delta^{2} / c^{2}\right) B \eta}{(1-K \eta)^{3}} \frac{d(c \kappa)}{d(x / c)}-\frac{K^{2}}{(1-K \eta)^{2}}-\frac{B R U}{1-K \eta}-R \frac{d V}{d \eta}\right] v \\
- & {\left[\frac{(\delta / c)}{1-K \eta} \frac{\partial V}{\partial(x / c)}+\frac{2 K R U^{2}}{1-K \eta}-\frac{2 B K}{(1-K \eta)^{2}}-\frac{\left(\delta^{2} / c^{2}\right)}{(1-K \eta)^{3}} \frac{d(c \kappa)}{d(x / c)}\right] u } \\
& =\frac{R}{2} \frac{d C_{\eta}}{d \eta}
\end{aligned}
$$

$$
\begin{aligned}
& \frac{d^{2} w}{d \eta^{2}}-\left[R V+\frac{K}{1-K \eta}\right] \frac{d w}{d \eta} \\
& +\left[\frac{(\delta / c)}{(1-K \eta)^{2}} \frac{d B}{d(x / c)}+\frac{B^{2}}{(1-K \eta)^{2}}-A^{2}+\frac{\left(\delta^{2} / c^{2}\right) B \eta}{(1-K \eta)^{3}} \frac{d(c \kappa)}{d(x / c)}-\frac{B R U}{1-K \eta}\right] w \\
& \quad=-\frac{A R C_{p}}{2}
\end{aligned}
$$

$$
\frac{B u}{1-K \eta}+\frac{d v}{d \eta}+A w-\frac{K v}{1-K \eta}=0 .
$$


It is desired that the analysis cover approximately the following range of variables.

$$
\begin{array}{cl}
K= & \delta \div \text { radius of curvature- }- \text { from } 0 \text { to } .01, \text { that is, to about } 30 \text { times the } \\
& \text { boundary layer thickness, } \\
B & =\text { dimensionless growth factor-from } 0 \text { to about } 0.2, \\
A & =\text { dimensionless measure of vortex size }- \text { from } 0.1 \text { to } 10 \\
R & =U_{0} \delta / \nu-\text { from about } 500 \text { to } \infty \\
c \kappa & =\text { airfoil chord } \div \text { radius of curvature }- \text { from } 0 \text { to } 10 \\
\frac{d(c \kappa)}{d(x / c)} \quad= & \text { dimensionless rate of change of curvature-from } 0 \text { to about } 200 \\
\frac{d B}{d(x / c)} \quad= & \text { dimensionless rate of change of } \beta-\text { from } 0 \text { to about } 10 .
\end{array}
$$

The range of $\mathrm{B}$ itself is not preselected; for $K$ is the independent variable. With the range chosen for $K$ this, as well as Görtler's analysis, indicates that $B$ will cover a range about as noted. In physical applications infinite rates of change of curvature, $d(c \kappa) / d(x / c)$ can easily be found. For instance, any discontinuity in curvature is a case; such a discontinuity exists at the pressure jump on a Griffith airfoil. At such places both $d\left(c_{\kappa}\right) /$ $d(x / c)$ and $d B / d(x / c)$ exceed the above noted ranges, and under such conditions, the equations to follow will be inadequate. It is not likely, however, that predictions of $\int \beta d x$ over a region of concave curvature will be seriously in error, for a strong rate of change of curvature by its very nature, must take place in a short distance.

Orders of magnitude of the various terms were studied carefully in the original work. A tremendous range of magnitudes was found. The largest terms are of the order of $R$, that is $O\left(10^{3}\right)$. The smallest terms are such as $K^{2}$ and $\left(\delta^{2} / c^{2}\right) B \eta d(c \kappa) / d(x / c)$. These terms are of order $<10^{-4}$ and prove to be of amazingly minor importance, proving that only the magnitude of the curvature is of significance. The fact that $K^{2}$ is of the same small order demonstrates that the system of equations can be treated as being essentially linear in $K$. Certain terms in the equations normally are small, yet at some place in the range they may become the major term. But, examination of the very, very smallest terms shows that at no time do they ever approach dominance; there always exists some other term that is far larger.

The simplified equations are presented below as the system (10) and (11). The only terms dropped are those affecting coefficients by less than $1 \%$ at all times, both for the full range of $B, K, A$ and $R$, and for $\eta$ less than about 10. To this point no mention of the term $1-K \eta$ has been made. If $\eta=10$ and $K=.01, K \eta=0.1$, a value as significant as many terms retained. In Görtler's analysis, the term, $1-K_{\eta}$, was assumed always to be equal to 1 . Consistent with the remainder of the analysis, such an approximation in the present case would not be satisfactory. Therefore, $(1-K \eta)^{-p}$ is replaced by the first term of its expansion: $1+p K \eta$. Then if $p=1$ and $K \eta=.1$, the coefficient is specified correctly within $1 \%$. The transformation not only is a valid approximation mathematically, but physically is likely to be an improvement. In the original development a singularity occurs at $K \eta=1$. Using the approximate expression, the singularity is removed to $\eta=\infty$. A $(\varphi-\psi)$ net of streamlines and equipotential lines outside the boundary layer will bear little resemblance to the grid system of Fig. 3. Instead, such a net will show a gradual merging into a rectangular system well away from the 
surface. A coordinate system in which the ordinates merge at $\eta=\infty$, instead of at $K_{\eta}=1$, appears actually to be nearer physical reality.

$$
\begin{aligned}
& \frac{d^{2} u}{d \eta^{2}}-(R V+K) \frac{d u}{d \eta}+\left[B^{2}-A^{2}-B R U+R \frac{d V}{d \eta}-K B R U_{\eta}\right] u \\
& -\left[R \frac{d U}{d \eta}-K R V\right] v=\frac{B R C_{\nu}}{2} \\
& \frac{d^{2} v}{d \eta^{2}}-(R V+K) \frac{d v}{d \eta}+\left[B^{2}-A^{2}-B R U-R \frac{d V}{d \eta}-K B R U \eta\right] v \\
& -2 K R U u=\frac{R}{2} \frac{d C_{\nu}}{d \eta} \\
& \frac{d^{2} w}{d \eta^{2}}-(R V+K) \frac{d w}{d \eta}+\left[B^{2}-A^{2}-B R U-K B R U \eta\right] w=-\frac{A R C_{p}}{2} \\
& \frac{B u}{1-K \eta}+\frac{d v}{d \eta}-\frac{K v}{1-K \eta}+A w=0 .
\end{aligned}
$$

In solving Eqs. (10) and (11) a certain boundary layer profile, $U(\eta)$, etc., must be specified. If the curvature is gentle, experiment and theory both show that the boundary layer is that predicted for the proper pressure gradients ignoring the curvature. It follows that if the pressure gradient is zero, regardless of the curvature, the boundary layer will be a Blasius profile. However, when the boundary layer thickness is perhaps $1 / 20$ of the radius of curvature, the free stream velocity is undergoing rapid changes, and the boundary profile may not be as expected. But in many cases, $\theta$, the momentum thickness, will be known with reasonable accuracy. Görtler has shown that the instability is mainly a function of $\theta$, not of details of the shape of the boundary layer. As a consequence, the equations should not break down prematurely just because the boundary layer shapes become unknown, provided $\theta$ is known.

10. Reduction of Eqs. (10) and (11) to a pair of simultaneous equations. Galerkin's method, used to find the eigenvalue $K$, requires solution of a large number of simultaneous algebraic equations. If Eqs. (10) and (11) were used as a basis, evaluation of coefficients and the like would be moderately brief, but the number of equations to solve would be excessive. When this system of equations is reduced to two, the time for setup of the simultaneous equations becomes slightly larger, but the number of equations to solve is only two-thirds as many. By this logic, reduction to a single sixth order equation would be best of all. Attempts were made, but unless drastic, accuracy-reducing simplifications are introduced, the equation becomes terribly long, so long that the increased setup time would more than nullify the time saved in solving the simultaneous equations. Hence, a solution beginning with two simultaneous differential equations is believed to require the minimum labor. To avoid introducing errors by the simplifications of the system (10) and (11), reduction was performed by continuing with system (6) and (7). The procedure is lengthy but direct. A system in $u$ and $v$ results. The equations were made non-dimensional and simplified in the fashion used for Eqs. (8) and (9). The resultant "simplified" equations are: 


$$
\left.\begin{array}{rl}
\left(\frac{B^{2}}{A^{2}}\right. & \left.-1+2 K \eta \frac{B^{2}}{A^{2}}\right) \frac{d^{2} u}{d \eta^{2}}+\left\{R V\left(1-\frac{B^{2}}{A^{2}}\right)+K\left[1+\frac{B^{2}}{A^{2}}-2 \eta R V \frac{B^{2}}{A^{2}}\right]\right\} \frac{d u}{d \eta} \\
& +\left\{B R U\left(1-\frac{B^{2}}{A^{2}}\right)-R \frac{d V}{d \eta}+A^{2}\left(1-\frac{B^{2}}{A^{2}}\right)^{2}+K\left[B R U \eta\left(1-\frac{3 B^{2}}{A^{2}}\right)\right.\right. \\
& \left.\left.-R V \frac{B^{2}}{A^{2}}\right]\right\} u \\
& +\left(\frac{B}{A^{2}}+\frac{K B \eta}{A^{2}}\right) \frac{d^{3} v}{d \eta^{3}}-\left[\frac{B R V}{A^{2}}+K\left(\frac{2 B}{A^{2}}+\frac{B \eta R V}{A^{2}}\right)\right] \frac{d^{2} v}{d \eta^{2}} \\
& -\left\{\frac{B^{2}}{A^{2}} R U+B\left(1-\frac{B^{2}}{A^{2}}\right)+K\left[B \eta\left(1-\frac{3 B^{2}}{A^{2}}\right)+\frac{2 B^{2}}{A^{2}} \eta R U-\frac{B}{A^{2}} R V\right]\right\} \frac{d v}{d \eta} \\
& +\left\{R \frac{d U}{d \eta}-K\left[R U\left(1-\frac{B^{2}}{A^{2}}\right)-2 B-B\left(1-\frac{B^{2}}{A^{2}}\right)\right]\right\} v=0
\end{array}\right\}
$$

$$
\begin{aligned}
\left(\frac{B}{A^{2}}\right. & \left.+K \eta \frac{B}{A^{2}}\right) \frac{d^{3} u}{d \eta^{3}}-\left[\frac{B R V}{A^{2}}+K\left(\frac{B \eta R V}{A^{2}}-\frac{2 B}{A^{2}}\right)\right] \frac{d^{2} u}{d \eta^{2}} \\
& -\left\{B\left(1-\frac{B^{2}}{A^{2}}\right)+\frac{B^{2}}{A^{2}} R U+\frac{B}{A^{2}} R \frac{d V}{d \eta}+K\left[B \eta\left(1-\frac{3 B^{2}}{A^{2}}\right)+2 \frac{B^{2}}{A^{2}} \eta R U^{2}\right.\right. \\
& \left.\left.+\frac{B \eta}{A^{2}} R \frac{d V}{d \eta}+\frac{2 B}{A^{2}} R V\right]\right\} \frac{d u}{d \eta} \\
& -\left\{\frac{B^{2}}{A^{2}} R \frac{d U}{d \eta}-K\left[2 R U\left(1-\frac{B^{2}}{A^{2}}\right)-2 \eta \frac{B^{2}}{A^{2}} R \frac{d U}{d \eta}-\frac{B}{A^{2}} R \frac{d V}{d \eta}\right]\right\} u \\
& +\frac{1}{A^{2}} \frac{d^{4} v}{d \eta^{4}}-\left(\frac{R V}{A^{2}}+\frac{2 K}{A^{2}}\right) \frac{d^{3} v}{d \eta^{3}}-\left\{\frac{B R U}{A^{2}}+\frac{R}{A^{2}} \frac{d V}{d \eta}+2-\frac{B^{2}}{A^{2}}\right. \\
& \left.+K\left[\frac{B R U \eta}{A^{2}}-\frac{R V}{A^{2}}\right]\right\} \frac{d^{2} v}{d \eta^{2}} \\
& +\left\{R V-\frac{B R}{A^{2}} \frac{d U}{d \eta}+K\left[\frac{R}{A^{2}} \frac{d V}{d \eta}-\frac{B \eta}{A^{2}} \frac{R d U}{d \eta}+2+\frac{B^{2}}{A^{2}}\right]\right\} \frac{d v}{d \eta} \\
& +\left\{B R U+R \frac{d V}{d \eta}+A^{2}\left(1-\frac{B^{2}}{A^{2}}\right)+K\left[B R U \eta+\frac{B}{A^{2}} R \frac{d U}{d \eta}\right]\right\} v=0
\end{aligned}
$$

If $B=0$ (Neutral Stability):

$$
\frac{d^{2} u}{d \eta^{2}}-(R V+K) \frac{d u}{d \eta}-\left(A^{2}-R \frac{d V}{d \eta}\right) u-\left(R \frac{d U}{d \eta}-K R U\right) v=0
$$

$2 K R U u+\frac{1}{A^{2}} \frac{d^{4} v}{d \eta^{4}}-\left[\frac{R V}{A^{2}}+\frac{2 K}{A^{2}}\right] \frac{d^{3} v}{d \eta^{3}}-\left[\frac{R}{A^{2}} \frac{d V}{d \eta}+2-\frac{K R V}{A^{2}}\right] \frac{d^{2} v}{d \eta^{2}}$

$$
+\left[R V+K\left(\frac{R}{A^{2}} \frac{d V}{d \eta}+2\right)\right] \frac{d v}{d \eta}+\left(R \frac{d V}{d \eta}+A^{2}\right) v=0
$$


11. Solution by Galerkin's method. Equations (12) can be regrouped as follows:

$$
\begin{aligned}
& \varphi_{1}(D) u+\varphi_{2}(D) v+K\left[\psi_{1}(D) u+\psi_{2}(D) v\right]=0, \\
& \varphi_{3}(D) u+\varphi_{4}(D) v+K\left[\psi_{3}(D) u+\psi_{4}(D) v\right]=0 .
\end{aligned}
$$

Since only $K$ to the first power occurs in the equations, $K$ is the most suitable coefficient to treat as the eigenvalue. $B$ or $A$ could so be treated except that they occur in the equations in a more complicated form. The problem then becomes one of finding the value of $K$ that meets the boundary conditions when $A, B, R$ and the boundary layer profile, $U(\eta)$ are specified. By physical considerations, it is obvious that the disturbance must decay in the free stream. Exactly on the surface there can be no velocity relative to the surface. Therefore, the six boundary conditions are

$$
\begin{aligned}
u(\infty) & =0, & u(0) & =0, \\
v(\infty) & =0, & v(0) & =0, \\
w(\infty) & =0, & w(0) & =0 .
\end{aligned}
$$

Furthermore, Eq. (11) shows that $d v / d \eta(0)=0$. This last feature may be called a secondary boundary condition. These homogeneous equations with homogeneous boundary conditions have been solved in the main by Galerkin's method. The method is an extremely powerful variation method, in many respects suggestive of the RayleighRitz method. See Ref. [8] for particulars; only the specific application will be treated in this study. For the present problem, because of the complexity and high order of the equations, six term solutions were used for most computations. They are

$$
\begin{aligned}
u & =e^{-E_{\eta}}\left[a_{1} \eta+a_{2} \eta^{2}+a_{3} \eta^{3}+a_{4} \eta^{4}+a_{5} \eta^{5}+a_{6} \eta^{6}\right], \\
v & =e^{-E_{\eta}}\left[b_{2} \eta^{2}+b_{3} \eta^{2}+b_{4} \eta^{4}+b_{5} \eta^{5}+b_{6} \eta^{6}+b_{7} \eta^{7}\right] .
\end{aligned}
$$

These automatically meet the six homogeneous boundary conditions. Furthermore (16b) meets the secondary condition, $d v / d \eta(0)=0$. The decay factor $E$ can be varied as necessary. If properly chosen, an accurate solution can be obtained. If improperly chosen, a poor solution is likely. $E$ is specified, leaving the $a_{i}$ and $b_{i}$ as unknowns to be determined.

In the present problem, because calculations were so lengthy, only the Blasius boundary layer was used. Values for it were taken from Ref. [9]. Boundary layer velocity ratios and the various derivatives were approximated to at least three decimal place accuracy by polynomial fits*. The various terms in the Galerkin equations then could be integrated analytically, for only expressions of the form $\eta^{p} e^{-2 E_{\eta}}$ were involved.

12. The matrix problem. Using the Galerkin procedure and expressions (16) twelve simultaneous algebraic equations are obtained, which in matrix algebra can be written:

$$
[P+K Q]\left\{a_{1}, a_{2}, a_{3}, a_{4}, a_{3}, a_{6}, b_{2}, b_{3}, b_{4}, b_{5}, b_{6}, b_{7}\right\}=0 \text {, }
$$

where $P$ and $Q$ are square matrices of the coefficients obtained from the Galerkin equations and \{\} represents a column matrix in the unknown coefficients. $P$ represents the matrix of coefficients determined from the $\varphi_{i}$ expressions, and $Q$ represents the values determined from the $\psi_{i}$ terms, Eq. (14).

${ }^{*} U, U^{\prime}, U^{\prime \prime}$ etc. were approximated independently; for example, the polynomial approximating $U$ was not differentiated to obtain $l^{\prime \prime}$. 
The primary interest is to find the mode of flow that will grow at the greatest rate along a wall of specified curvature. If, as in actual calculations, $B$ is prescribed, the problem is to find the least concave curvature that will generate that degree of instability. Since several modes of flow exist, the problem becomes one of finding the least value of $K$, that is, the largest value of $1 / K$, assuming all roots are positive. Accordingly, Eq. (17) is treated in the form

$$
\left[\frac{1}{K} P+Q\right]\left\{a_{1}, a_{2}, \cdots, a_{6}, b_{2}, b_{3}, \cdots, b_{7}\right\}=0 .
$$

Then the roots $1 / K$ are given by the characteristic matrix

$$
S=P^{-1} Q \text {. }
$$

The items of major interest are, first, the lowest value of $K$, and second, the accompanying modal column-the eigenfunction. Because of the high order of the matrix, the iterative method was chosen as being the least laborious. It will converge on the largest value of $|1 / K|$.

13. Accuracy and miscellaneous notes concerning the calculations. Table I lists all cases solved. Most cases were computed using the six-term approximations (16). Approximations using fewer terms were obtained by dropping the highest powers of $\eta$ in (16). Inspection will show several convergence studies distributed throughout the field which throw light on the accuracy.

The most complete study of convergence was made at $A=1, B=0$. The very first calculations were made by the method of collocation, Sec. 7.9, Ref. [10]. In this method the error $\epsilon$ in a fitted expression is made exactly zero at several points. The collocation method is to Galerkin's method as Lagrangian interpolation is to least squares curve fitting. Ref. [10] states that it is less accurate than Galerkin's method. The present work bears out the statement, for the Galerkin values are always closer to the most exact value when the number of terms in the approximation expressions are equal. The six-term values obtained by both methods agree well, however. The results of the other convergence studies should be clear from the table.

Because the present analysis includes terms neglected by Görtler, the equations cannot be reduced to expressions in terms of his parameters, and computations must be made for specific values of $R$. Nevertheless, parameters similar to his so predominate that it is convenient to use them. As in his analysis, one parameter is $R_{\theta}(\theta / r)^{1 / 2}$, or in the coordinate system of Eqs. (12), $R K^{1 / 2}$. The other dominant parameter is $B R$ which if referred to momentum thickness would be $\beta \theta R_{\theta}$. Görtler's similar parameter was $\beta \theta^{2} / \nu$. Now as $\beta \theta R_{\theta} \equiv \beta \theta^{2} U_{0} / \nu$ the two differ by a velocity, which follows as a consequence of Görtler's use of a timewise growth of the vortices. To verify that the two parameters $R K^{1 / 2}$ and $B R$ are dominant, two pairs of cases were checked: $A=.1$, $B=0, R=2000$ vs $A=.1, B=0, R=6000$, and $A=3, B=.05, R=4000$ vs $A=3, B=.1, R=2000$. If these indeed were the only parameters, then the same value of $R K^{1 / 2}$ should be obtained for either Reynolds number. The values agree well.

Careful review of all convergence studies made indicates that the individual points appear correct within about two per cent, but such accuracy probably does not hold throughout the final chart, Fig. 5, for the reason that too few points were computed to define well the various curves. The central portions of each curve in Fig. 5 may have errors up to 10 per cent, the extremities even more. 
TABIE 1

Summary of Results

\section{A}

.03

.10

.10

.10

.10

.10

.10

.10

.10

.30

.30

.30

.30

.30

1.0

1.0

1.0

1.0

1.0

1.0

1.0

1.0

1.0

1.0

1.0

1.0

1.0

3.0

3.0

3.0

3.0

10.0

10.0

10.0

10.0

10.0

10.0

$B$
.01

0
0
0
0
0
.01
.10
.10

$R$

2000

2000

2000

2000

2000

6000

2000

2000

2000

2000

2000

2000

2000

2000

2000

2000

2000

2000

2000

2000

2000

2000

2000

2000

2000

2000

2000

.20

0

.05

.05

.10

2000

2000

4000

2000

2000

2000

2000

2000

2000

2000

\section{E}

2

2

2

1

0.5

0.5

2

2

2

2

1

2

2

2

2

2

2

2

2

2

2

2$$
2
$$

2

2

2

2

2

2

2

2

2

2

2

2

2

2
No. Terms

in

Approximation $\quad R K^{1 / 2}$

Galerkin

575.6

5. 10

1.353

1.430

0.998

0.996

186.3

1513.5

890.5

1.143

1.096

40.54

232.8

500.7

6.530

3.411

2.873

2.865

2.857

1.072

2.624

3.026

2.821

125.3

241.6

241.5

481.1

16.05

102.5

180.3

178.6

162.7

156.5

246.7

327.7

324.7

465.8

The disturbance functions, $u, v, w$, in many cases, are much less accurate. Some cases, by fortuitous choice of $E$, appear superficially to be good solutions. Others obviously are poor representations of the disturbance. Nevertheless, in spite of the doubtful accuracy of many of the eigenfunctions, most solutions are presented in Figs. 6, 7 and 8, 


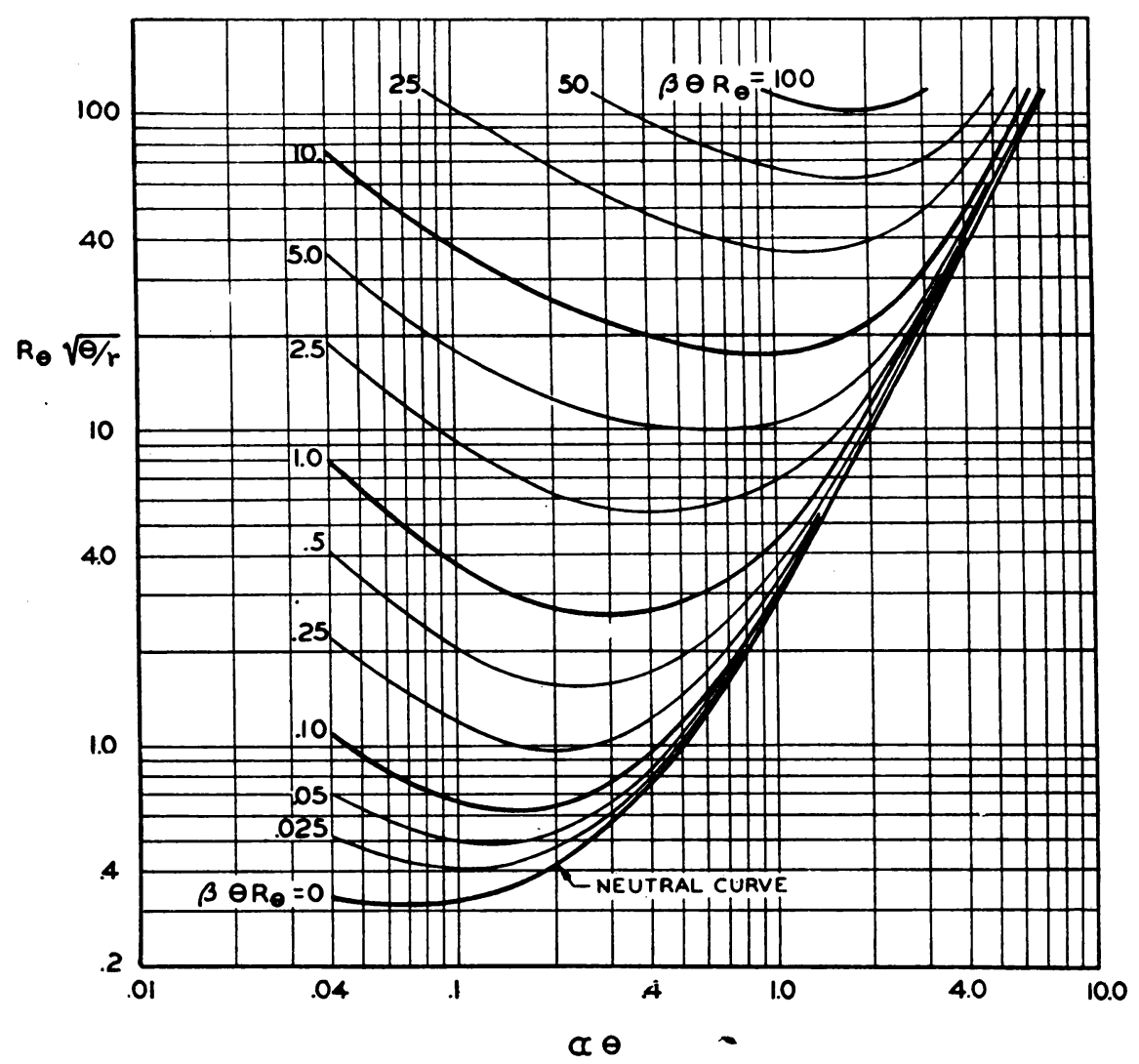

FIG. 5. Stability of Taylor-Görtler vortices.

where some impression of the accuracy may be obtained by comparing the several cases. Further discussion of these functions will be reserved for Sec. 15, Results.

14. A second method of solution. The excessive labor and difficult matrices created a desire to find a faster method of solution-one having improved accuracy if possible. A promising method was found. In any problem of boundary layer flow in free air, the boundary conditions exist at $\eta=0$ and $\eta=\infty$. But a boundary layer extends only a slight way from $\eta=0$; beyond it lies the free stream, which may be treated as a region of constant velocity in the direction of $\eta$, even on an airfoil. In this region $U=1$, $d U / d \eta=0$, etc. Consequently, for problems in boundary layer flow, the disturbance equations simplify to ones in which all important transcendental type of coefficients such as $U(\eta)$ disappear or else become constant coefficients. This fact, together with a matrix interpolation procedure, forms the basis for the method presented on the following pages. The method is far more rapid than the Galerkin method provided a large scale digital computer can be used. Otherwise, it is too slow to be considered as an alternate.

We shall solve the pair of equations (12), although the method would be just as applicable to the original set (10). Equations (12) are a homogeneous pair forming a sixth order system containing an unknown constant. The boundary conditions are homogeneous. In the previous method $K$ was chosen as the unknown because, to the same order of approximation, only $K$ to the first power occurred; whereas $B$, for instance, 
E• 2 EXCEPT AS NOTED

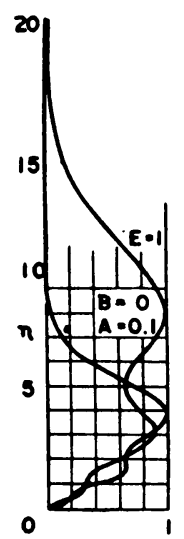

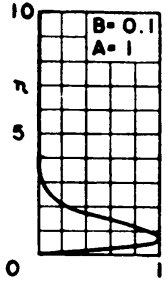
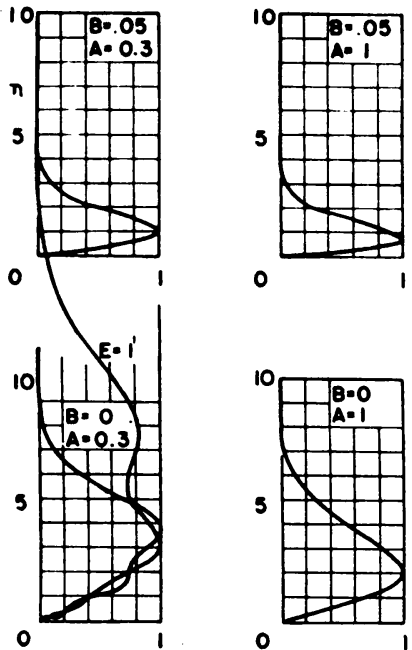
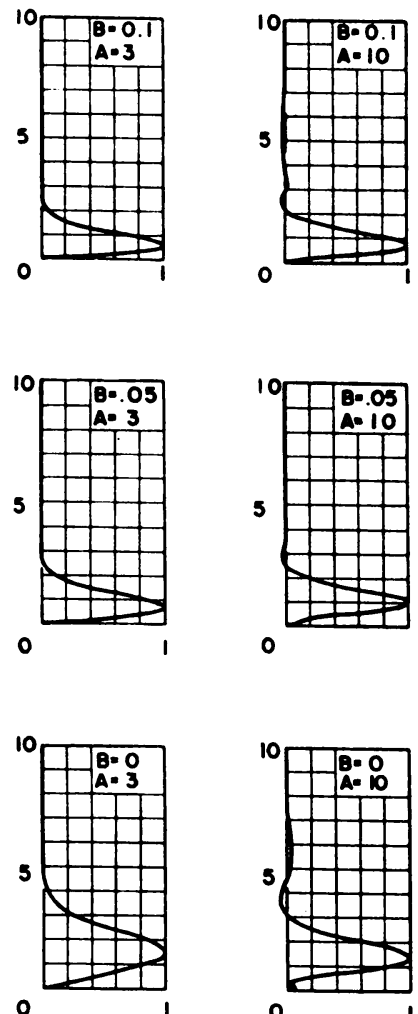

FIG. 6. Nature of the $u$-component of the disturbance.

occurred in powers as high as $B^{4}$. In this new analysis any one of the constants $K, B, A$, or $R$ could be treated as the unknown. However, $K$ is retained as the unknown, partly because it is convenient and partly because the previous work has been along this line.

The general system is linear but has variable coefficients of a transcendental type, which, however, may be approximated satisfactorily for all practical purposes by simple polynomials. Any homogeneous system of order $n$ has $n$, and only $n$, independent solutions, independence being tested by the Wronskian. For the present, assume $K$ is known. Then when six such solutions are found, the complete solution meeting all boundary conditions can be formed. The key to the six can be found by solving the system (12) outside the boundary layer, for beyond the boundary layer, especially the Blasius case

$$
R V=\text { Constant }=R V_{0} ; R d V / d \eta=0 ; U=1 ; d U / d \eta=0 .
$$

In the special case $\beta=0$ and beyond the boundary layer, the equations reduce to the following pair containing only constant coefficients.

$\frac{d^{2} u}{d \eta^{2}}-\left(R V_{0}+K\right) \frac{d u}{d \eta}-A^{2} u+K R v=0$,

$2 K R u+\frac{1}{A^{2}} \frac{d^{4} v}{d \eta^{4}}-\left(\frac{R V_{0}}{A^{2}}+\frac{2 K}{A^{2}}\right) \frac{d^{3} v}{d \eta^{3}}-\left(2-\frac{K R V_{0}}{A^{2}}\right) \frac{d^{2} v}{d \eta^{2}}$

$$
+\left(R V_{0}+2 K\right) \frac{d v}{d \eta}+A^{2} v=0
$$



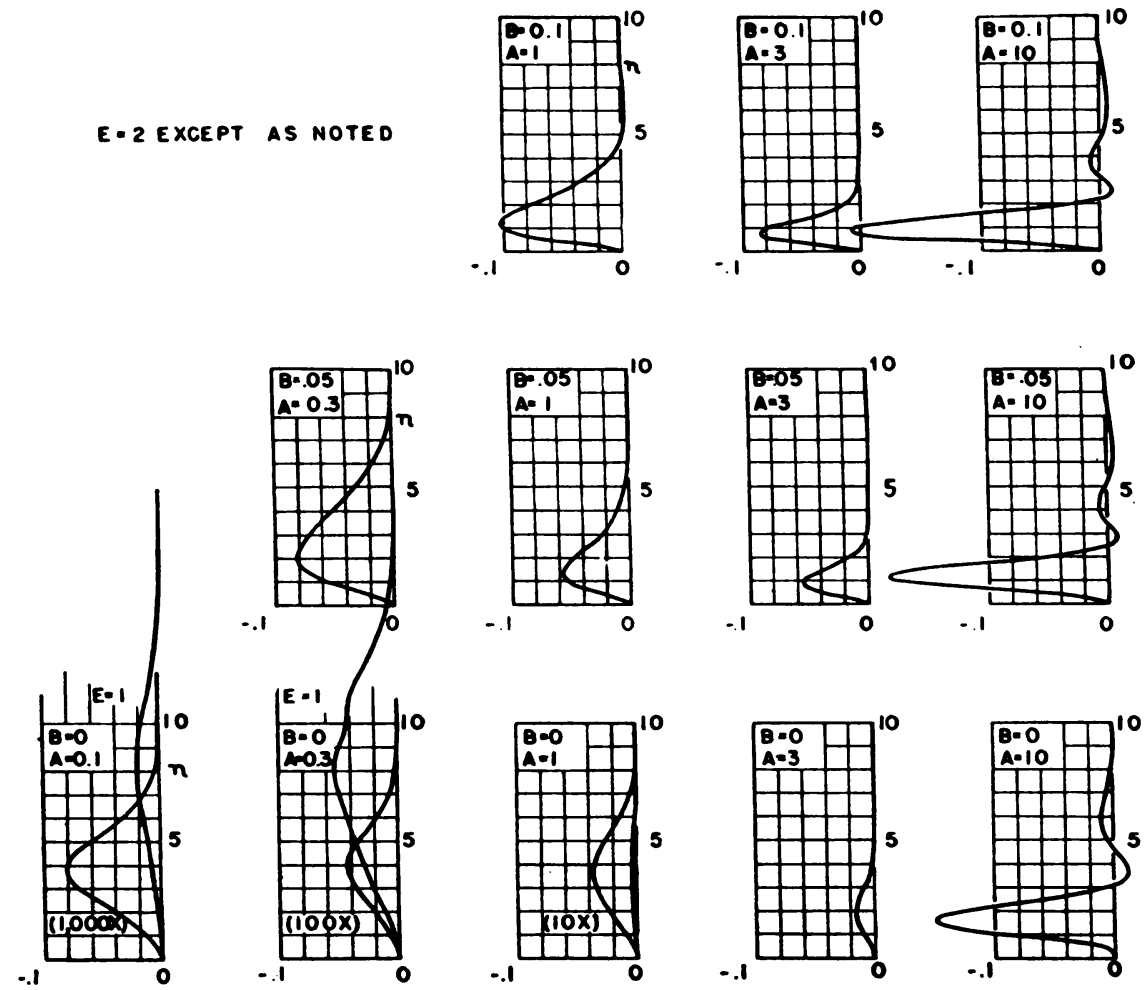

Fig. 7. Nature of the $v$-component of the disturbance.

Outside the boundary layer, Eqs. (12) can be written in the shorter form

$$
\begin{aligned}
\left(M_{1}+N_{1} K \eta\right) \frac{d^{2} u}{d \eta^{2}} & +\left(M_{2}+N_{2} K \eta\right) \frac{d u}{d \eta}+\left(M_{3}+N_{3} K \eta\right) u+\left(M_{4}+N_{4} K \eta\right) \frac{d^{3} v}{d \eta^{3}} \\
& +\left(M_{5}+N_{5} K \eta\right) \frac{d^{2} v}{d \eta^{2}}+\left(M_{6}+N_{6} K \eta\right) \frac{d v}{d \eta}+M_{i} v=0 \\
\left(P_{1}+Q_{1} K \eta\right) \frac{d^{3} u}{d \eta^{3}} & -\left(P_{2}+Q_{2} K \eta\right) \frac{d^{2} u}{d \eta^{2}}-\left(P_{3}+Q_{3} K \eta\right) \frac{d u}{d \eta}+P_{4} u+P_{5} \frac{d^{4} v}{d \eta^{4}} \\
& -P_{6} \frac{d^{3} v}{d \eta^{3}}-\left(P_{7}+Q_{7} K \eta\right) \frac{d^{2} v}{d \eta^{2}}+P_{8} \frac{d v}{d \eta}+\left(P_{9}+Q_{9} K \eta\right) v=0
\end{aligned}
$$

in which $M_{i}, N_{i}, P_{i}, Q_{i}$ are constants as indicated by Eqs. (12).

Consider the special case $\beta=0$, (Eq. 19). Six solutions of (19) are readily found, provided $K$ is specified. The solutions obviously are elementary functions, obtained by computing the roots of the sixth degree auxiliary equation. Outside the boundary layer, the general solution of (19) can be written

$$
u \text { or } v=c_{1} \varphi_{1}+c_{2} \varphi_{2}+\cdots+c_{6} \varphi_{6}
$$

where the $\varphi_{i}$ are the independent solutions. The $c_{i}$ differ, of course, according to whether the solutions are for $u$ or $v$. Because of the way these solutions have been obtained, there 

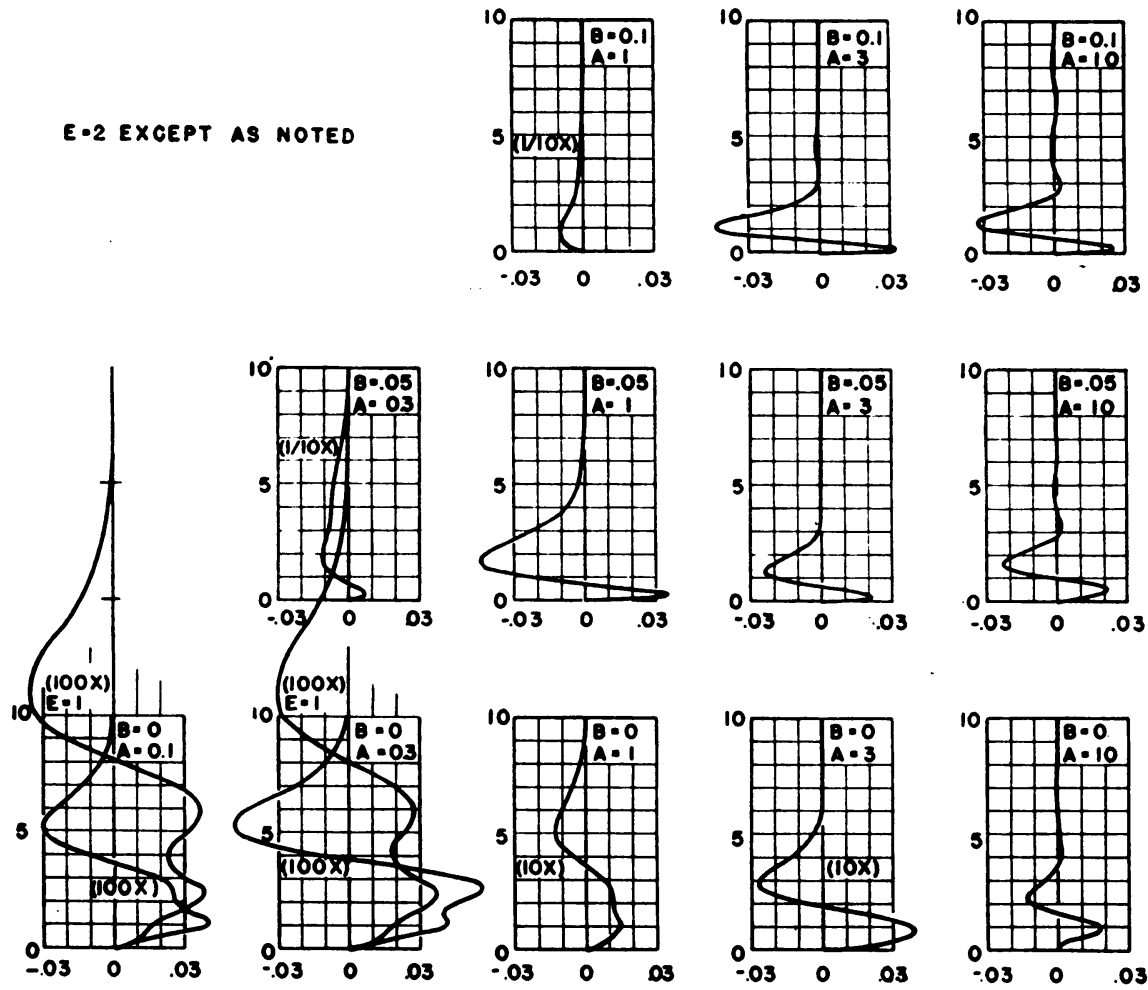

FIG. 8. Nature of the $w$-component of the disturbance.

is no question of independence. The general solution then for neutral stability is seen to be given in terms of elementary functions for all the region outside the boundary layer. Each complete solution can now be extended through the boundary layer to $\eta=0$ by means of numerical integration methods. The boundary layer shape can easily be represented with high accuracy. The numerical extension requires only a few minutes on a large scale digital computer. On an IBM Card Programmed Calculator about twenty hours are necessary for each solution $\varphi_{i}$ if steps small enough to obtain about four-figure accuracy are used.

Return now to the general case (20). For it, numerical computation within the boundary layer differs only in the number of factors, not in nature. If all initial conditions are clearly specified, there are no problems of uniqueness of the solution within the boundary layer. For consider the general differential equation.

$$
p_{0}(x) \frac{d^{n} y}{d x^{n}}+p_{1}(x) \frac{d^{n-1} y}{d x^{n-1}}+p_{2}(x) \frac{d^{n-2} y}{d x^{n-2}}+\cdots+p_{n}(x) y=0
$$

If the variable coefficients $p_{0}, p_{1}$, etc., are continuous, single valued functions of $x$ only, throughout an interval $a \leq x \leq b$ and if $p_{0}$ does not vanish at any point in the interval, then there exists a unique continuous solution $y(x)$ which assumes a value of $y_{0}$ at a point $x_{0}$ within $(a, b)$ and whose first $n-1$ derivatives are continuous and assume respectively, the values $y_{0}^{\prime}, y_{0}^{\prime \prime}, \cdots, y_{0}^{n-1}$ at $x_{0}$. The differential equations (12) have been examined by tracing through what happens to their coefficients when reduced to 
a single equation in $u$ or $v$. Within the boundary layer all the conditions stated above are met. The term equivalent to $p_{0}$, the coefficient of $d^{6}() / d \eta^{6}$ in the single equation, definitely remains finite in the complete range. Beyond the boundary layer, the solutions are of a different nature due to the existence of the terms $K \eta$. This part of the coefficient of each derivative is small at the joining point because $\eta$ is $<10$, and because of the smallness of $K$ itself $(<.01$ ). Its small value with respect to the constant term suggests a method of successive approximations, beginning with solutions given by Eqs. (20), but with the terms involving $K_{\eta}$ neglected. One such procedure has been briefly investigated and found suitable although others requiring less labor may well be found.

Within the range of interest it is known that any solution $\varphi_{i}$ of (20) may be expressed in a uniformly convergent series in powers of $K$, a small parameter, Ref. [11], Sec. 3.31.

$$
\varphi_{i}=\varphi_{i n}+K \varphi_{i 1}+K^{2} \varphi_{i 2}+K^{3} \varphi_{i 3}+\cdots+K^{r} \varphi_{i r}+\cdots \quad(i=1,2, \cdots, 6) .
$$

Because the general system (12) cannot easily be reduced to a single equation, it is convenient to deal in the six paired values $u_{i}$ and $v_{i}$, or

$$
\begin{aligned}
& u_{i}=u_{i 0}+K u_{i 1}+K^{2} u_{i 2}+K^{3} u_{i 3}+\cdots+K^{r} u_{i r}+\cdots, \\
& v_{i}=v_{i 0}+K v_{i 1}+K^{2} v_{i 2}+K^{3} v_{i 3}+\cdots+K^{r} v_{i r}+\cdots,
\end{aligned}
$$

where each $u_{i r}, v_{i r}$ is a function of $\eta$. Substitute these expressions into (20) and collect terms according to powers of $K$. An infinite series of linear differential equations is obtained which may be solved in succession to obtain each additional term in (23). Symbolically, the system may be written

$$
\begin{aligned}
& L_{1}\left(u_{i 0}\right)+L_{2}\left(v_{i 0}\right)=0 \\
& L_{i 3}\left(u_{i 0}\right)+L_{4}\left(v_{i 0}\right)=0 \\
& L_{1}\left(u_{i 1}\right)+L_{2}\left(v_{i 1}\right)=\eta\left[M_{1}\left(u_{i 0}\right)+M_{2}\left(v_{i 0}\right)\right] \\
& L_{3}\left(u_{i 1}\right)+L_{4}\left(v_{i 1}\right)=\eta\left[M_{3}\left(u_{i 0}\right)+M_{4}\left(v_{i 0}\right)\right] \\
& L_{1}\left(u_{i 2}\right)+L_{2}\left(v_{i 2}\right)=\eta\left[M_{1}\left(u_{i 1}\right)+M_{2}\left(v_{i 1}\right)\right] \\
& L_{3}\left(u_{i 2}\right)+L_{4}\left(v_{i 2}\right)=\eta\left[M_{3}\left(u_{i 1}\right)+M_{4}\left(v_{i 1}\right)\right] \\
& \left(\text { for } K^{-0}\right) \\
& \text { (a) } \\
& \text { (for } K^{1} \text { ) } \\
& \text { (for } K^{2} \text { ) }
\end{aligned}
$$

Solutions $u_{i 0}, v_{i 0}$ are six in number and involve only the constant part of the coefficients in Eq. (20). They in turn are used in the right-hand side of (24b) which then provides new solutions $u_{i 1}, v_{i 1}$. The operators on the left are always the same. Hence, the first correction term reduces to one of determining the particular integral.

Repeated roots never occur in any case of interest. Therefore, the only solutions possible from (24a) are of the type exp $(\lambda \eta)$, exp $(\gamma \eta) \cos \mu \eta, \exp (\gamma \eta) \sin \mu \eta$. Examination of successive solutions for the particular integrals, by the method of undetermined coefficients, shows that every additional term in the series (23) always contains the same exponential. Thus, if the first approximation, $u_{i 0}, v_{i 0} \rightarrow 0$ as $\eta \rightarrow \infty$, any and all successive terms will likewise approach zero. Therefore, it is possible to select solutions capable of meeting boundary conditions $u(\infty), v(\infty), v^{\prime}(\infty)=0$, with the certainty that a higher order term in the series will never cause the series to diverge. 
In recapitulation, when $B=0$, initial conditions for the numerical extension of the solutions are given by elementary functions obtained by solving a pair of equations having constant coefficients. When $B \neq 0$, initial conditions can be provided by the solutions (23) of (20).

Of the six independent solutions, only ones having negative exponentials are admissible if the boundary conditions at $\eta=\infty$ are to be met, for no combination of functions involving different positive exponentials will reach a value zero at $\eta=\infty$. Routh's method has been used to examine the signs of roots of the auxiliary equation derived from (24a), that is, of the first approximation. In all cases examined, representing the range of interest, three roots had positive real parts. Therefore, three solutions can be dropped; and for the present problem, Eq. (21) can be written

$$
u \text { or } v=c_{1} \varphi_{1}+c_{2} \varphi_{2}+c_{3} \varphi_{3} \text {. }
$$

For example, in the free stream $\varphi_{1}$ may be $\exp (-\gamma \eta) \cos \mu \eta$, and $\varphi_{2}$ may be $\exp (-\gamma \eta)$ $\sin \mu \eta$. If $u_{1}$ corresponds to $\exp (-\gamma \eta) \cos \mu \eta$, then $v_{1}$ corresponding will be a combination of $\varphi_{1}$ and $\varphi_{2}$, as determined by substitution of $u_{1}$ in the free stream equations. With these three remaining solutions, all boundary requirements at $\eta=\infty$ are automatically met. For any set of values of $B, A, K, R$ and boundary layer profile $U(\eta)$, numerical extension of the three remaining free stream solutions will provide the following information:

Solution

$\begin{array}{cccc}\varphi_{1} & u_{10} & v_{10} & v_{10}^{\prime} \\ \varphi_{2} & u_{20} & v_{20} & v_{20}^{\prime} \\ \varphi_{3} & u_{30} & v_{30} & v_{30}^{\prime}\end{array}$

The boundary conditions require that $u(0)=v(0)=v^{\prime}(0)=0$. Therefore, if $q_{i}$ are constants

$$
\begin{aligned}
& q_{1} u_{10}+q_{2} u_{20}+q_{3} u_{30}=0 \\
& q_{1} v_{10}+q_{2} v_{20}+q_{3} v_{30}=0 \\
& q_{1} v_{10}^{\prime}+q_{2} v_{20}^{\prime}+q_{3} v_{30}^{\prime}=0
\end{aligned}
$$

In general, this system will be incompatible unless $K$ is chosen correctly. $K$, however, is an unknown to be found. Assume that the approximate value of $K$ is known, as would be true from previous calculations if a $K-A-B$ plot were being constructed. Since $K$ is known approximately, choose two values for $K$, preferably bracketing the expected value. Let them be identified as $K_{1}, K_{2}$. Compute all six admissible solutions for these two values of $K$. At $\eta=0$, intercepts as follows can be read from the results of the numerical integrations.

Solution

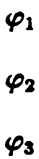

Values at $\eta=0$

$\begin{array}{lllllll}\varphi_{1} & \overbrace{{ }_{1} u_{10}} & { }_{1} v_{10} & { }_{1} v_{10}^{\prime} & \overbrace{{ }_{2} u_{10}} & { }_{2} v_{10} & { }_{2} v_{10}^{\prime} \\ \varphi_{2} & { }_{1} u_{20} & { }_{1} v_{20} & { }_{1} v_{20}^{\prime} & { }_{2} u_{20} & { }_{2} v_{20} & { }_{2} v_{20}^{\prime} \\ { }_{1} u_{30} & { }_{1} v_{30} & { }_{1} v_{30}^{\prime} & { }_{2} u_{30} & { }_{2} v_{30} & { }_{2} v_{30}^{\prime}\end{array}$


If the variation of these values with $K$ is approximately linear, interpolation formulae for each of the nine values can be written, e.g.,

$$
u_{10}={ }_{1} u_{10}+\frac{\left({ }_{1} u_{10}-{ }_{2} u_{10}\right)}{K_{1}-K_{2}}\left(K-K_{1}\right) .
$$

Let $\sigma$ represent the proportionality factor $\left({ }_{1} u_{10}-{ }_{2} u_{10}\right) /\left(K_{1}-K_{2}\right)$ and let $K-$ $K_{1}=\Delta K$. Then for $u_{10}$ we can write

$$
u_{10}={ }_{1} u_{10}+\sigma_{11} \Delta K .
$$

When all values (27) are used to determine similar relations, Eqs. (26) may be written

$$
\begin{aligned}
& \left.q_{1}\left({ }_{1} u_{10}+\sigma_{11} \Delta K\right)+q_{2}\left({ }_{1} u_{20}+\sigma_{12} \Delta K\right)+q_{3}{ }_{1} u_{30}+\sigma_{13} \Delta K\right)=0, \\
& q_{1}\left({ }_{1} v_{10}+\sigma_{21} \Delta K\right)+q_{2}\left({ }_{1} v_{29}+\sigma_{22} \Delta K\right)+q_{3}\left({ }_{1} v_{30}+\sigma_{23} \Delta K\right)=0, \\
& q_{1}\left({ }_{1} v_{10}^{\prime}+\sigma_{31} \Delta K\right)+q_{2}\left({ }_{1} v_{20}^{\prime}+\sigma_{32} \Delta K\right)+q_{3}\left({ }_{1} v_{30}^{\prime}+\sigma_{33} \Delta K\right)=0,
\end{aligned}
$$

or in matrix form

$$
\left[\begin{array}{ccc}
{ }_{1} u_{10} & { }_{1} u_{20} & { }_{1} u_{30} \\
{ }_{1} v_{10} & { }_{1} v_{20} & { }_{1} v_{30} \\
{ }_{1} v_{10}^{\prime} & { }_{1} v_{20}^{\prime} & { }_{1} v_{30}^{\prime}
\end{array}\right]\left[\begin{array}{l}
q_{1} \\
q_{2} \\
q_{3}
\end{array}\right]+\Delta K\left[\begin{array}{ccc}
\sigma_{11} & \sigma_{12} & \sigma_{13} \\
\sigma_{21} & \sigma_{22} & \sigma_{23} \\
\sigma_{31} & \sigma_{32} & \sigma_{33}
\end{array}\right]\left[\begin{array}{l}
q_{1} \\
q_{2} \\
q_{3}
\end{array}\right]=0 .
$$

That is, in short hand notation

$$
[P+\Delta K Q] q=0 .
$$

Equation (28) is a cubic in $\Delta K$ which may be solved by obtaining the equation explicitly, or by iteration as before for Galerkin's method. If the proper value of $K$ has been bracketed well, the desired root will be the smallest value of $\Delta K$. Otherwise, all roots should be examined. Once the value of $K=K_{1}+\Delta K$ is found, a new set of three runs should be made, based on this new-found value, to serve as a check run or to obtain an improved approximation.

Using advanced digital computors, the method is rapid, very accurate, and quite flexible, for either $B, A, K$ or $R$ can be used as the eigenvalue. Furthermore, the method permits the unknown constant to occur in any form. The interpolation procedure will be subject to the same difficulties that beset Newton's method of determining the zeros of a polynomial. Thus, cases may arise where the interpolated $K$ given by (29) may be worse than the earlier trial value. However, such difficulties are not expected to be any more serious than they are in Newton's method. A further drawback of the subject procedure is that it tells nothing about the number and location of other roots, for it obtains only one at a time. If applied to an entirely new problem, exploratory work by other less accurate methods may be desirable.

An example. Only one case has been solved by this method, that for $A=1, B=0$, $R=2000$. Solutions were computed for $K=.00001$, and .000002 . By Galerkin's method, the correct value of $K$ is $\mathbf{0 0 0 0 0 2 0 4}$. Hence, one value is at some distance from the correct value; the other is very close. The three admissible free stream solutions are

$$
\varphi_{1}=e^{-\omega \eta}, \quad \varphi_{2}=e^{-\lambda \eta} \cos \mu \eta, \quad \varphi_{3}=e^{-\lambda \eta} \sin \mu \eta .
$$


For three values of $K$ the usable roots are

K

.0001

.00001

.000002 $\omega$

1.0235829

1.0002648

1.0000098 $\lambda$

.55527247

.56205005

.56213472 $\mu$

.14151315

.014604736

.0029290325

The free stream solutions were extended by the Taylor's series method of extrapolation, working directly with Eqs. (13). The joining point chosen as the edge of the boundary layer was at $\eta=5.7$ corresponding to $U=1.00000$.

Computations were performed on the IBM Card Programmed Calculator using increments of $\eta=.01$ or .02 , according to the nature of the higher derivatives. A few convergence checks using other values of $\Delta \eta$ indicated the solutions were accurate to about three significant figures. By this method, each run requires about twenty hours on the Card Programmed Calculator, that is, 120 hours of computation must be done before a minimum set of values is available for the interpolation procedure. For this reason the runs were mostly of exploratory nature to serve both as a check on the theory back of this second method and to serve as an overall check of Galerkin's method.

15. Results. Because of the great labor involved; a chart for only one Reynolds number-boundary layer combination was prepared. The boundary layer was the Blasius type; the Reynolds number $R$ was 2000, corresponding to $R_{0}=U_{0} \theta / \nu=939$. Crossplots of $R K^{1 / 2} v s$. $B$ for constant values of $A$ conveniently proved to be nearly straight lines, greatly facilitating interpolation. Recommended interpolation polynomials, representing these cross-plots, are listed in Table 2.

Table 2.

Interpolation formulae for $R K^{1 / 2}$ as a function of $B R$ and $A . R=2000$, Blasius boundary layer

\section{$A$}

0.1

0.3

1.0

3.0

10.0
Formula

$$
R K^{1 / 2}=.998+4.4475 B R
$$$$
R K^{1 / 2}=1.096+1.8581 B R+.00598(B R)^{2}-.0000139(B R)^{3}
$$$$
R K^{1 / 2}=2.82+1.278 B R-.000638(B R)^{2}+.000001081(B \dot{R})^{3}
$$$$
R K^{1 / 2}=16+.9175 B R-.000525(B R)^{2}
$$

$R K^{1 / 2}=156.4+.983 B R-.000895(B R)^{2}+.000000927(B R)^{3}$

Note: For above case, $\beta \theta R_{\theta}=(.4696)^{2} B R, \alpha \theta=.4696 A$

$$
R_{\theta}(\theta / r)^{1 / 2}=(.4696)^{3 / 2} R K^{1 / 2}
$$

Görtler's work showed the solutions to be primarily a function of $\theta$, the momentum thickness, the actual shape of the boundary layer being found to have only a secondary influence on the results. Therefore, from the cross-plot data, a chart in terms of $\theta$, similar to his, has been constructed, and is presented as Fig. 5. By its use, growth of nearly any size vortex along concave surfaces of any shape can easily be computed simply by 
evaluating $\int_{x}^{x_{2}} \beta d x$. The chart is most accurate at $R=2000$ and for the Blasius boundary layer, but as discussed earlier, the parameters used in the plot enable it to be used for other conditions with only a small loss of accuracy. The neutral curve agrees roughly with Görtler's neutral curve. Görtler computed the minimum value of the neutral stability curve to be $R_{\theta}(\theta / r)^{1 / 2}=0.58$; in the present work the value is about 0.32 . Meksyn's minimum value was 3.65. Difference between the present and Görtler's values may be attributed to three factors:

(a) The present analysis includes the effect of boundary layer growth, that is, $R V \neq 0$.

(b) For a given order matrix, Galerkin's method should supply more precise eigenvalues than those given by Green's functions and Fredholm's method of solving the resulting integral equations.

(c) Higher order matrices were used in the present study-twelfth order vs. eighth for Görtler.

Factors $(b)$ and $(c)$ are believed to account for most of the disagreement.

Plots of the $u, v$ and $w$ components of disturbances are presented in Figs. 6, 7 and 8 . The $u$ disturbance has arbitrarily been given a maximum value of unity; the $v$ and $w$ components then are presented in their true proportions to the $u$ component. The component $w$ is computed by means of Eq. (9).

Accuracy of these disturbance functions is quite uncertain. Undoubtedly there is a wide variation in accuracy depending on how well the decay factor $\exp \left(-E_{\eta}\right)$ was chosen. Certain solutions appear especially poor, those for $A=0.1$ and 0.3 at $B=0$ being good examples. In these two cases the two sets of curves represent two decay factors $\exp (-\eta)$ and $\exp (-2 \eta)$. The oscillations in the curves near $\eta=0$ are believed to be imposed by the poor fit of the Galerkin expression; physically, no such oscillation should happen. Probably a still lower value of $E$ would smooth out the curve. Nevertheless, in spite of the considerable disagreement in the shape of these two solutions, the eigenvalue, $K$, was nearly the same for both. The central group of solutions are expected to have the highest accuracy.

In spite of the uncertain accuracy, the family of plots is informative, for it indicates the approximate height of the disturbance and shows roughly how the height and shape vary with $A$ and $B$. One of the most notable features is the very strong increase of the $v$ and $w$ components with $A$; at low values of $A$ there is almost no $v$ and $w$ component. The disturbance plots are useful for throwing light on the energy balance, but no detailed study of such has been made.

Figure 1 shows two vortex patterns for $A=1$ and $R=2000$; one is for $B=0$, the other for $B=0.1$. These have been computed by the method of isoclines using the $v$ and $w$ components to indicate the flow direction at any point.

The six-term Galerkin solution and the second method produced the following values for $K$ :

$$
\left.\begin{array}{ll}
K=2.041 \times 10^{-6} & \text { By six-term Galerkin } \\
K=1.989 \times 10^{-6} & \text { By second method }
\end{array}\right\} A=1, B=0, R=2000
$$

Consequently, the two values of $R_{\theta}(\theta / r)^{1 / 2}$ will agree within two per cent. Figure 9 presents the eigenfunctions according to both methods. Because in this case $u$ is by far the strongest component, values of $u$ by the two methods agree rather well. The $v$ solution 


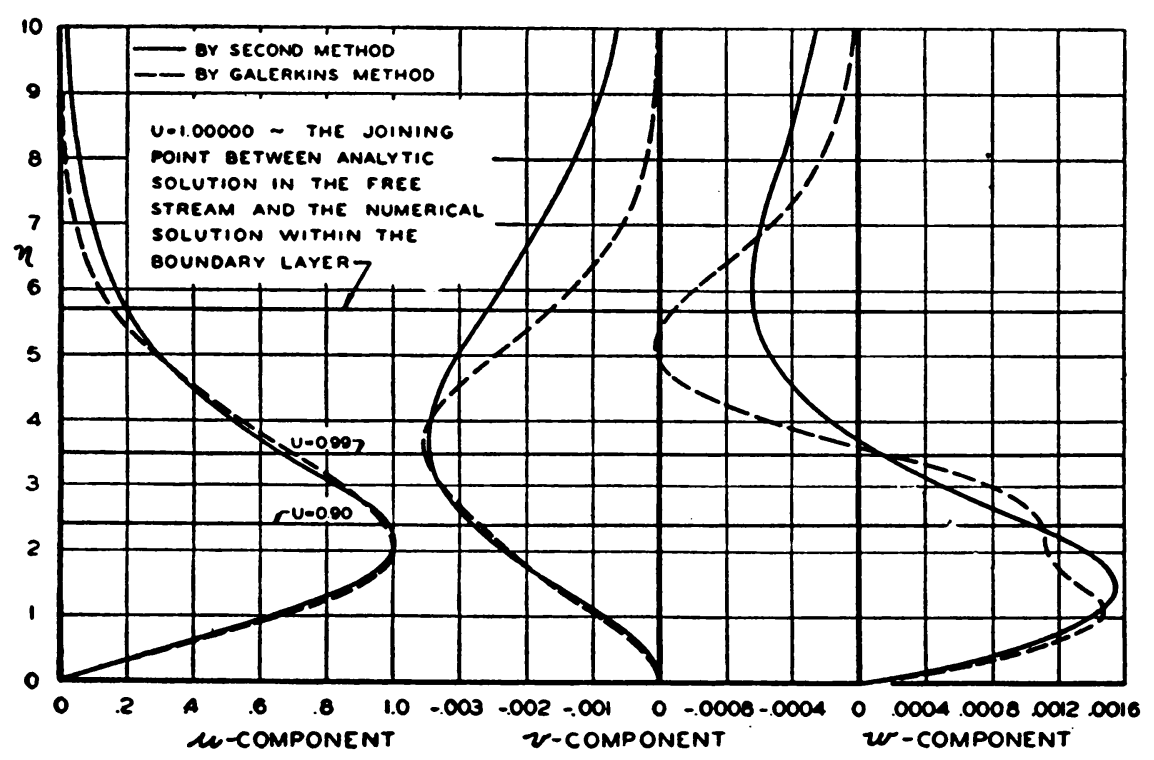

Fig. 9. The disturbance functions according to two methods of calculation.

agrees just as well, but because its magnitude is much less, the percentage accuracy is considerably worse. Since $w$ does not enter directly into the solution and since with $B=0, A=1, w \doteq-d v / d \eta$ slight errors in $v$ will be magnified by $w$.

Earlier, with regard to solutions for $B=0, A=0.1$ and 0.3 , the odd shape of the $u$ component solutions in Fig. 6 was attributed to the mathematics, for physically such a mode should not exist. The plots of the $w$ component, on Fig. 9, bear out this explanation, for the considerably more accurate solution by the second method does not show the irregularity indicated by the less accurate Galerkin solution.

16. The prediction of transition and agreement with experiment. When a fluid moves past a flat or convex surface, transition of the ordinary type will occur. That is, wavelike disturbances begin and grow, due to Tollmien-Schlichting instability, surface roughness, and turbulence, until the fluctuations are so large that turbulent flow begins to form. When the flow is along a wall having concave curvature, Görtler vortices will develop and grow provided the curvature and boundary layer Reynolds number are sufficiently great. If the curvature is slight, the ordinary type of wave growth will occur, but if it is large, Görtler instability will so predominate that the transition point will be determined entirely by this type of flow.

The actual mechanics of this second type of transition are unknown, since a steady vortex by itself would not necessarily cause transition. Possibly the vortex modifies the boundary layer profile sufficiently to make it unstable to two-dimensional waves. Perhaps, as suggested by Liepmann [5], transition occurs when the apparent shear arising from a disturbance reaches a certain ratio to the basic laminar shear. If so, even though the rate of growth of the vortices is substantially a function of $\theta$ only, the actual boundary layer profile can still influence the transition point. For if the apparent shear must reach a certain ratio to the laminar shear, then a more stable profile will require a stronger vortex to maintain the ratio. Certainly it can be said that regardless of the mechanics 
of transition, the vortices must attain a certain strength with respect to a characteristic velocity before transition can occur.

In lieu of detailed knowledge of the true development of turbulence, the next best method of determining the effect of concave curvature is to compute, as well as possible, the growth of the vortices, which by some unknown means do cause transition when they become strong enough. That is, since experiment has shown that these vortices have some connection with transition on concave surfaces, the best method of predicting their effect is to follow their growth, as well as possible, to the very point of transition and then to correlate with experiment.

The motion that can be developed by these vortices on plates of variable curvature in a growing boundary layer can be most complex. As conditions change, Fig. 5 shows that of the infinitude of possible vortices, one size will grow strongest, only to lose its ascendancy to one of a different size. All wave-lengths can exist simultaneously. The motion that can be developed may bear little resemblance to that of only a single vortex.

In spite of the possibilities of having a heterogeneous conglomeration of vortices of all sizes and strengths, each and every one obeys the predictions of Fig. 5, provided it is weak. Furthermore, within the limits of the assumption that disturbances are small, the linear nature of the differential equations shows that these disturbances of any wave-length are additive. Therefore, the best means available for following the growth of vortices and their subsequent evolution into turbulent flow appears to be one of evaluating $\int \beta d x$ from the point of neutral stability onward. Various wave-lengths as measured by $\alpha$ are tried until the one maximizing $\int \beta d x$ is found, for the maximum value of $\int \beta d x$ is being sought. By so doing, we determine the factor $\exp \int \beta d x$, which indicates the ratio of the strength of the disturbance to its strength at the neutral stability point. What must truly determine transition is the magnitude of these vortices. But exp $\int \beta d x$ is not a magnitude, it is a ratio. To know magnitude one must know the magnitude of the disturbances at the neutral point. Lack of such knowledge constitutes a weak point in the argument for use of $\int \beta d x$ as a criterion. Undoubtedly, the magnitude at the neutral point is related to the magnitude of the turbulence in free stream as well as to waves in the boundary layer. Furthermore, the spectrum of the stream turbulence must be considered; certainly the ordinary turbulence value is an inadequate measure, for Liepmann's tests [5] on the effect of turbulence show that the product $\left(u^{\prime} / U\right) \exp \int \beta d x$ at transition is not a constant.

Nevertheless, when dealing with wind tunnels of about the same level of turbulence, the available experimental data show $\exp \int \beta d x$ at transition to be a better parameter than any used in the past. The results are shown in Fig. 10. The only data available are from the experiments noted on the figure. One set is by Liepmann, obtained from tests on plates of constant curvature [5] in a special tunnel. The velocity along the plate was constant. His investigation covered the case of a growing boundary layer on plates of constant curvature.

A quite different type of test was performed in the small Douglas Wind Tunnel. Transition was determined on the concave side of a $30^{\prime \prime}$ chord, 1/4" thick, 24ST aluminum alloy plate curved to the NACA $a=1, C_{L_{D_{e s}}}=1$ camber line. The turbulence in this tunnel is low as noted with the data points. In this tunnel, at the test angle of attack of four degrees, the velocity along the concave side is accurately defined by the expression $U / U_{\infty}=.806(x / c)^{.0545} ; .01 \leq x / c \leq .70$. In these tests, of course, the radius of curvature varied greatly along the chord. 


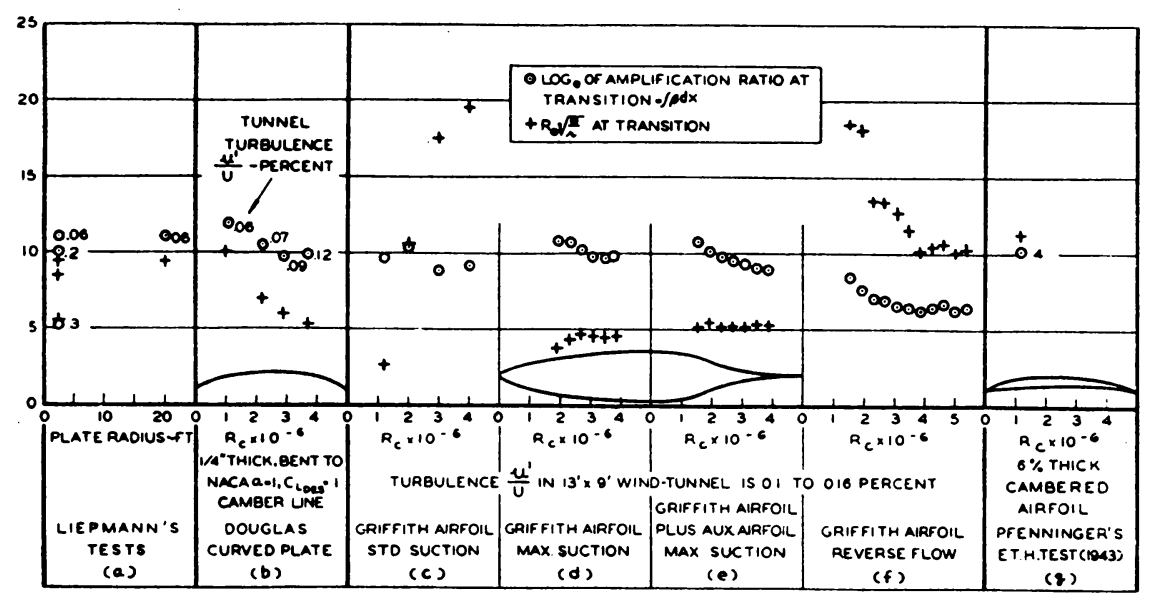

FIr. 10. Summary of experimental data on Görtler vortex type of transition.

A third experiment was performed in England while the flow behind the slot on a $16 \%$ Griffith airfoil was being examined [12]. Four types of tests were run: one with the normal type of flow and suction, a second with suction so strong as to remove nearly all the boundary layer, a third with the same strong suction but with a velocity distribution modified by means of an auxiliary airfoil, and finally a test with the airfoil facing backwards.

The fourth experiment, conducted by W. Pfenninger at the E.T.H. in Zurich, represents an incidental observation made during tests of a cambered propeller section. The velocity along the concave side was substantially constant back to the observed transition point.

A few comments about each of the tests will now be made. As was to be expected from the theory, Liepmann found that transition occurred at the same value of $R_{\theta}(\theta / r)^{1 / 2}$ for either radius of curvature. Both of his tests were for similar flows, that is, constant radius of curvature, constant free stream velocity and a boundary layer that began at the leading edge of the plate. When such conditions apply, the rate parameter $R_{\theta}(\theta / r)^{1 / 2}$ also is a measure of the strength of the vortices at transition. Hence, as he verified, $R_{\theta}(\theta / r)^{1 / 2}$ is a satisfactory indicator of transition on dimensionally similar flows. In addition, Liepmann's data provide a few valuable clues concerning the effect of tunnel turbulence. However, the rough measure of magnitude of the vortices, $\left(u^{\prime} / U\right) \exp \int \beta d x$, is not even approximately constant for the three turbulence levels.

Curvatures of the Douglas curved plate varied from $c / r=3.5$ to 0.4 to 3.5 from the leading edge to midchord to trailing edge, respectively. To a first approximation; the values of $\int \beta d x$ for the most critical wave-length agree with Liepmann's tests. Probably some of the change in $\int \beta d x$ with Reynolds number is attributable to changes in the turbulence level noted on the figure, for the trends are in the proper direction.

The next test is a very interesting one, because it represents the case of flow behind a pressure jump on a Griffith airfoil. The general level of the values agrees closely with that of the first two tests. Therefore, the slot itself does not appear to cause a premature transition. Furthermore, the slightly lower level and negative slope of the points can be accounted for partially by the increased turbulence level of this tunnel and by the 
effect of speed on turbulence. In the next test, maximum suction was applied in order to remove all the boundary layer so that a fresh boundary layer would develop behind the slot.

On the normal airfoil, the flow from the slot to the trailing edge is accelerating considerably. In order to learn the effect of velocity distribution, the British mounted an auxiliary airfoil nearby to create an essentially constant velocity along this region behind the slot. Figures (10d) and (10e) show that the change had little effect on $\int \beta d x$.

Furthermore, these two figures indicate a distinct downward trend of $\int \beta d x$ with Reynolds number. In Ref. [12], it is stated that the blower had capacity sufficient to remove the entire boundary layer only at the lower Reynolds numbers. At the maximum Reynolds number only about three-fourths was being removed. Any allowance for such initially thicker boundary layer aft of the slot would appreciably increase $\int \beta d x$ at the transition point. Some crude calculations indicate $\int \beta d x$ would increase by the order of 0.5 if allowance were made for the thicker boundary layer.

The final Griffith airfoil test represents a reverse flow experiment on the same airfoil. For some unknown reason, the general level of $\int \beta d x$ is appreciably less than that for the five other tests. Possibly in such a peculiar test, the boundary layer received outside disturbances. Certainly the flow on the opposite side of the airfoil was separated.

The parameters $R_{\theta}(\theta / r)^{1 / 2}$ or a weighted mean of $R_{\theta}(\theta / r)^{1 / 2}$ to account for variable curvature have been suggested in the past as indicators of transition. Figure 10 shows that $R_{\theta}(\theta / r)^{1 / 2}$ is entirely inadequate; values vary by nearly a tenfold factor. In one experiment, they reduce as a function of Reynolds number; in another, the values increase depending on the distribution of curvature, boundary layer thickness, and boundary layer Reynolds number. Moreover, in several cases, the value of $R_{\theta}(\theta / r)^{1 / 2}$ at transition was much lower than values encountered upstream prior to transition where the curvature happened to be greater.

Any weighted mean value of $R_{\theta}(\theta / r)^{1 / 2}$ likewise seems doubtful as a valid indicator of transition. If the flow passes a short depression, the curvature may be so high that any type of mean value of $R_{\theta}(\theta / r)^{1 / 2}$ over this region would be very large. Yet, the length may be so low that transition will fail to occur. Length of the region should be included to obtain a proper weighting.

Evidently at first, when the vortices are weak and turbulence is low, the flow along a concave wall can be described physically by the methods of this report. In particular the strength grows with distance just as does $\int \beta d x$. However, as the vortices become strong, the mathematics no longer describes the motion. But, in spite of a divergence of the mathematical description and physical reality, computation of $\int \beta d x$ at transition appears to be sufficiently close to the actual events to provide a usuable indicator of the point of transition.

\section{REFERENCES}

1. S. Goldstein, Modern developments in fluid dynamics, vol. 1, Clarendon Press, Oxford, 1938

2. Iord Rayleigh, On the dynamics of revolving fluids, Proc. Roy. Soc. (London) A(93), $148-154$ (1917)

3. G. I. Taylor, Stability of a viscous liquid contained between two rotaling cylinders, Phil. Trans. Roy. Soc. (London) A(223) 289-343 (1923)

4. H. Görtler, Über eine dreidimensionale Instabilität laminarer Grenzschichten an konkaven Wänden, Ges. d. Wiss. Göttingen, Nachr. a. d., Math., 2, No. 1 (1940)

5. H. W. Liepmann, Investigation of boundary layer transition on concave walls, NACA Wartime Report ACR No. 4J28, Feb. 1945 
6. W. W. Hagerty, l'se of an optical property of glycerine-water solutions to study viscous fluid-flow problems, J. Appl. Mech. 17, 54-58 (1950)

7. D. Meksyn, Stability of viscous flow over concave cylindrical surfaces, Proc. Roy. Soc. (London) A(203), 253-265 (1950)

8. W. J. Duncan, Galerkin's method in mechanics and differential equations, British Reports and Memoranda No. 1798, (1937)

9. A. M. O. Smith, Improved solutions of the Falkner and Skan boundary layer equation, Sherman Fairchild Fund Paper No. FF-10, Inst. Aeronaut. Sci. (1954)

10. R. A. Frazer, W. J. Duncan, A. R. Collar, Elementary matrices, Cambridge University Press, Cambridge, 1950

11. E. L. Ince, Ordinary differential equations, Dover Publications

12. E. J. Richards, W. S. Walker, J. R. Greening, Tests of a Griffith Aerofoil in the $13 \mathrm{ft} . \times 9 \mathrm{ft}$. wind hinnel, British Reports and Memoranda No. 2148, March 1944

A personal communication from the N.P.L. supplied much additional and more detailed data than available in the $R \& M$. Furthermore, the factor 1.36 , page 9 of $R \& M 2148$ was changed to the correct value 0.54

13. Günther Hämmerlin, Über das Eigenwertproblem dreidimensionalen Instabilität laminarer Grenzschichten längs konkaven Wänden, J. Ratl. Mech. Anal. 4, 279-321 (1955). 